\title{
Knot Floer homology and integer surgeries
}

\author{
PETER S OZSVÁTH \\ ZOLTÁN SZABÓ
}

\begin{abstract}
Let $Y$ be a closed three-manifold with trivial first homology, and let $K \subset Y$ be a knot. We give a description of the Heegaard Floer homology of integer surgeries on $Y$ along $K$ in terms of the filtered homotopy type of the knot invariant for $K$. As an illustration of these techniques, we calculate the Heegaard Floer homology groups of non-trivial circle bundles over Riemann surfaces (with coefficients in $\mathbb{Z} / 2 \mathbb{Z}$ ).
\end{abstract}

57M27; 57M25

\section{Introduction}

Heegaard Floer homology is an invariant for closed-oriented three-manifolds $Y$ (see Ozsváth-Szabó [16]). The invariant, denoted $H F^{\circ}(Y)^{1}$, is the homology of a chain complex whose generators have a combinatorial definition, and whose boundary operator counts certain pseudo-holomorphic disks in associated spaces. Moreover, if $W: Y_{1} \longrightarrow Y_{2}$ is a smooth, connected, oriented cobordism from the connected, oriented three-manifold $Y_{1}$ to $Y_{2}$, equipped with a $\operatorname{Spin}^{c}$ structure, there is an associated map of Heegaard Floer homology groups. Explicit calculations of the chain complex and associated chain maps are difficult to make in general, though under favorable circumstances, the homology groups can be determined with the help of a long exact sequence relating the Heegaard Floer homologies of various surgeries on a given knot, cf Ozsváth-Szabó [15].

In Ozsváth-Szabó [14] and Rasmussen [21], a closely related invariant is defined for null-homologous knots $K$ in a closed, oriented three-manifold $Y$, taking the form of an induced filtration on the Heegaard Floer complex of $Y$. The filtered chain homotopy type of this complex is a knot invariant, to which we refer loosely as "knot Floer homology".

\footnotetext{
${ }^{1}$ In [16], we defined several variants of Heegaard Floer homology : $\widehat{H F}, H F^{-}, H F^{\infty}$, and $H F^{+}$, which are related to one another by various exact sequences. We denote this entire collection here by $H F^{\circ}$. In this paper, however, we will focus primarily on the case of $H F^{+}$.
} 
If $K \subset Y$ is null-homologous, there is a canonical identification of framings on $K$ with integers. Given an integer $n$, let $Y_{n}(K)$ denote the three-manifold obtained by $n$-framed surgery on $Y$ along $K$. When $n$ is sufficiently large, there is an immediate relationship between the knot Floer homology of $K$ and the Heegaard Floer homology of $Y_{n}(K)$, see [14;21]. In cases where $Y$ is sufficiently simple - for example, when $Y \cong S^{3}$ - these data are sufficient to determine $\mathrm{HF}^{+}\left(Y_{n}(K)\right)$ for arbitrary integers $n$. However, even this case, one loses information on some of the additional structure carried by Heegaard Floer homology (for example, its absolute grading).

The aim of this paper is to describe completely the Heegaard Floer homology of integral surgeries on $Y$ along $K$ in terms of data associated to the knot $K$, in the case where $Y$ has trivial first homology. To describe this construction, we recall some aspects of knot Floer homology.

Knot Floer homology associates to a knot $K$ in $Y$ a $\mathbb{Z} \oplus \mathbb{Z}$-filtered $\mathbb{Z}[U]$-complex $C=C F K^{\infty}(Y, K)$. More precisely, $C$ is generated over $\mathbb{Z}$ by a set $X$ equipped with a function $\mathcal{F}: X \longrightarrow \mathbb{Z} \oplus \mathbb{Z}$ with the property that if $\mathbf{x} \in X$ is an element with $\mathcal{F}(\mathbf{x})=(i, j)$, then $\partial \mathbf{x}$ can be written as a linear combination of elements $\mathbf{y} \in X$ with $\mathcal{F}(\mathbf{y}) \leq \mathcal{F}(\mathbf{x})$. Moreover, the action of $U$ on $C$ is induced from an action of $U$ on the generating set $X$, with the property that for each $\mathbf{x} \in X$ with $\mathcal{F}(\mathbf{x})=(i, j)$, then $\mathcal{F}(U \cdot \mathbf{x})=(i-1, j-1)$.

We write the two factors of the $\mathbb{Z} \oplus \mathbb{Z}$ filtration as $(i, j)$. Let $S$ be a region in the plane which has the property that for all $(i, j) \in S$ and all $\left(i^{\prime}, j^{\prime}\right) \geq(i, j)$, we have that $\left(i^{\prime}, j^{\prime}\right) \in S$. Then the subset of $C$ generated by points with filtration level contained in $S$ naturally inherits the structure of a quotient complex, which we write as $C\{S\}$. For example, $C\{i \geq 0\}$ denotes the quotient complex of $C$ generated by $\mathbf{x} \in X$ with $\mathcal{F}(\mathbf{x})=(i, j)$ with $i \geq 0$. There is a canonical (up to sign) chain homotopy equivalence $C\{i \geq 0\} \simeq C\{j \geq 0\}$. Indeed, $B^{+}=C\{i \geq 0\}$ is identified with $C F^{+}(Y)$.

Define $A_{s}^{+}=C\{\max (i, j-s) \geq 0\}$. There are two canonical chain maps $v_{s}^{+}: A_{s}^{+} \longrightarrow$ $B^{+}$and $h_{s}^{+}: A_{s}^{+} \longrightarrow B^{+}$. The map $v_{s}^{+}$is projection onto $C\{i \geq 0\}$, while $h_{s}^{+}$is projection onto $C\{j \geq s\}$, followed by the identification with $C\{j \geq 0\}$ (induced by multiplication by $\left.U^{s}\right)$, followed by the chain homotopy equivalence from $C\{j \geq 0\}$ to $C\{i \geq 0\}$.

Let $\mathbb{A}^{+}=\bigoplus_{s \in \mathbb{Z}} A_{s}^{+}$and $\mathbb{B}^{+}=\bigoplus_{s \in \mathbb{Z}} B_{s}^{+}$(for this latter group, each summand is isomorphic to $B^{+}$, but we include a subscript to distinguish the various summands), and let $D_{n}^{+}: \mathbb{A}^{+} \longrightarrow \mathbb{B}^{+}$be the map

$$
D_{n}^{+}\left(\left\{a_{s}\right\}_{s \in \mathbb{Z}}\right)=\left\{b_{s}\right\}_{s \in \mathbb{Z}},
$$


where here

$$
b_{s}=h_{s-n}^{+}\left(a_{s-n}\right)+v_{s}^{+}\left(a_{s}\right) .
$$

Let $\mathbb{X}^{+}(n)$ denote the mapping cone of $D_{n}^{+}$; ie this is the chain complex whose underlying group is $\mathbb{A}^{+} \oplus \mathbb{B}^{+}$, and whose differential over $\mathbb{Z} / 2 \mathbb{Z}$ has the form

$$
\left(\begin{array}{cc}
\partial_{\mathbb{A}}^{+} & 0 \\
D_{n}^{+} & \partial_{\mathbb{B}}
\end{array}\right)
$$

The following result says that the above data associated to the knot $K \subset Y$ contains enough information to deduce the Floer homologies of all the three-manifolds obtained by integer surgeries on $K$, and all the corresponding maps induced on homology by the natural cobordisms.

Theorem 1.1 Let $Y$ be an integral homology three-sphere. For any non-zero integer $n$, the homology of the mapping cone $\mathbb{X}^{+}(n)$ of

$$
D_{n}^{+}: \mathbb{A}^{+} \longrightarrow \mathbb{B}^{+}
$$

is isomorphic to $\mathrm{HF}^{+}\left(Y_{n}(K)\right)$. Moreover, under this identification the natural map $H F^{+}(Y) \cong H_{*}\left(B_{s}^{+}\right) \longrightarrow H_{*}\left(\mathbb{X}^{+}(n)\right)$ is identified with the map $H F^{+}(Y) \longrightarrow$ $H F^{+}\left(Y_{n}(K)\right)$ induced by the natural two-handle cobordism from $Y$ to $Y_{n}(K)$ endowed with the $s$ th $\operatorname{Spin}^{c}$ structure (for some identification of these $\operatorname{Spin}^{c}$ structures with $\mathbb{Z}$ ).

We have chosen our hypotheses of Theorem 1.1 to simplify the statement. For generalizations and refinements, see below. In particular, see Theorem 4.1 for a more precise statement of the isomorphism, which takes gradings into account, and Theorem 4.2, which makes explicit the identification of the maps induced by cobordisms. See also Section 4.8 for a discussion of the case where $n=0$, and Section 4.10 for further generalizations of Theorem 1.1. We return to the more general case of rational surgeries in the sequel (Ozsváth-Szabó [17]).

Note that knowing the filtered chain homotopy type of the knot filtration uniquely determines the groups $A_{s}^{+}$and $B_{s}^{+}$, and also the maps $v_{s}^{+}$. The maps $h_{s}^{+}$are, in general, not known explicitly, as they involve the chain homotopy equivalence $C\{i \geq 0\} \simeq$ $C\{j \geq 0\}$. Despite this shortcoming, Theorem 1.1 is still quite helpful in performing explicit calculations, as we shall see in Section 5. It is also worth noting that there is an overall \pm 1 ambiguity in the homotopy equivalence, and hence in the maps $h_{s}^{+}$; however, it is easy to see that the homology of the resulting complex is independent of this ambiguity. 
In Section 2, we recall some of the aspects of Heegaard Floer homology used later, and also set up some notation. In Section 3, we prove an exact sequence relating Heegaard Floer homology groups of $Y_{n}(K), Y_{m+n}(K)$, and $Y$, which is an ingredient in the proof of Theorem 1.1. In Section 4, we state and prove a more precise version of Theorem 1.1 (cf Theorem 4.1 and Theorem 4.2 below), taking into account the gradings on Floer homology. In Section 5, we give some sample calculations to illustrate the techniques from this paper. As an example, we calculate the reduced Heegaard Floer homology of any non-trivial circle bundle over a Riemann surface (with coefficients in $\mathbb{Z} / 2 \mathbb{Z})$.

Theorem 1.1, of course, strengthens the connection between Floer homology for knots and Floer homology for closed three-manifolds. This could be further pursued from several angles. For example, this result could be viewed as motivation for studying knot invariants in gauge-theoretic contexts, such as Seiberg-Witten or Donaldson's theories (compare Collin-Steer [2]). In a different direction, there seems to be a close connection between knot Floer homology and Khovanov's homology for links (cf Khovanov [4], Khovanov-Rozansky [5], Bar-Natan [1] and Lee [7]), see also recent work of Rasmussen [22]. It is an open problem whether Khovanov's homology admits an extension to a three-manifold invariant.

\section{Acknowledgements}

The authors wish to thank Tomasz Mrowka, Jacob Rasmussen, and András Stipsicz for valuable conversations during the course of this work. We are especially grateful to Slaven Jabuka and Thomas Mark for some very useful correspondence regarding the calculations of circle bundles.

PSO was supported by NSF grant number DMS 0505811.

ZS was supported by NSF grant number DMS 0406155.

\section{Review}

The purpose of this section is to introduce notation, recall some elements of Heegaard Floer homology used later, and also to put in place various preliminary notions. In Section 2.1, we set up terminology on gradings which we will use throughout this paper; in Section 2.2, we recall some standard terminology from homological algebra; in Section 2.3 we set up notation for the Heegaard Floer complexes; in Section 2.4, we set up notation and conventions for integral surgeries on null-homologous knots; in Section 2.5 we recall the relationship between knot Floer homology and Heegaard 
Floer homology of surgeries with sufficiently large (integral) coefficients (compare [14] and [21]); in Section 2.6 we verify Theorem 1.1 for the unknot in $S^{3}$. This calculation will be useful to us in the proof of Theorem 1.1. Finally, in Section 2.7, we include some simple observations about the $\mathbb{Z}[U]$-modules which we encounter in this paper.

\subsection{Gradings}

Let $C$ be a free $\mathbb{Z}$ module which is freely generated by some set $X$. We say that $C$ is relatively $\mathbb{Z}$-graded if there is a function

$$
\Delta: X \times X \longrightarrow \mathbb{Z}
$$

with the property that

$$
\Delta(x, y)+\Delta(y, z)=\Delta(x, z) .
$$

An element of $C$ is said to be homogeneous if it can be written as a linear combination of elements from a subset $S \subset X$ with the property that for all $x, y \in S, \Delta(x, y)=0$. An endomorphism $f: C \longrightarrow C$ is said to be homogeneous of degree $d$ if the image of any homogeneous element of $C$ is homogeneous, and indeed for all $x \in X, f(x)$ can be written as a linear combination of elements $y$ with $\Delta(y, x)=d$. The same terminology can be used when the relative grading takes values in the rational numbers, rather than the integers (in which case we call it a relative $\mathbb{Q}$-grading). A relatively graded chain complex is a relatively graded group $C$, which is equipped with a differential

$$
\text { д: } C \longrightarrow C
$$

which is homogeneous of degree -1 .

Let $C$ be a free $\mathbb{Z}$-module which is freely generated by some set $X$. We say that $C$ is absolutely graded if $X$ is equipped with a function

$$
\text { gr: } X \longrightarrow \mathbb{Q} \text {. }
$$

An absolute grading gr, of course, induces a relative $\mathbb{Q}$-grading by the formula

$$
\Delta(x, y)=\operatorname{gr}(x)-\operatorname{gr}(y) .
$$

In this case, the absolute grading gr is said to be a lift of the relative grading $\Delta$. If $C_{1}$ and $C_{2}$ are graded chain complexes, then a map $f: C_{1} \longrightarrow C_{2}$ is said to be homogeneous of degree $c$ if it carries homogeneous elements in $C_{1}$ with degree $a$ to homogeneous elements in $C_{2}$ with degree $a+c$. 


\subsection{Chain complexes}

We will often use standard notions from homological algebra, which we collect here for the reader's convenience.

Let $f: C_{1} \longrightarrow C_{2}$ be a chain map between $\mathbb{Z} / 2 \mathbb{Z}$-graded chain complexes. Then, the mapping cone $M(f)$ is the chain complex whose underlying group is $C_{1} \oplus C_{2}$, endowed with a differential

$$
(a, b) \mapsto\left(\partial a, \partial b+(-1)^{\operatorname{gr}(a)} \cdot f(a)\right) .
$$

There is a short exact sequence of chain maps

$$
0 \longrightarrow C_{2} \stackrel{\iota}{\longrightarrow} M(f) \stackrel{\pi}{\longrightarrow} C_{1} \longrightarrow 0,
$$

whose induced connecting homomorphism is identified (up to sign) with the map on homology induced by $f$,

$$
F_{*}: H_{*}\left(C_{1}\right) \longrightarrow H_{*}\left(C_{2}\right)
$$

Two maps $f, f^{\prime}: C_{1} \longrightarrow C_{2}$ are chain homotopic if there is a map $h: C_{1} \longrightarrow C_{2}$ with $\partial \circ h-h \circ \partial=f-f^{\prime}$. Two chain complexes $C_{1}$ and $C_{2}$ are said to be chain homotopy equivalent if there are chain maps $\phi: C_{1} \longrightarrow C_{2}$ and $\psi: C_{2} \longrightarrow C_{1}$ so that $\phi \circ \psi$ and $\psi \circ \phi$ are chain homotopic to the respective identity maps. The maps $\phi$ and $\psi$ are called chain homotopy equivalences. Chain homotopic maps give rise to chain homotopy equivalent mapping cones.

Let $C_{1}$ and $C_{2}$ be a pair of chain complexes. A quasi-isomorphism is a chain map $f: C_{1} \longrightarrow C_{2}$ which induces an isomorphism in homology. Of course, a chain homotopy equivalence is a quasi-isomorphism. Two complexes $C_{1}$ and $C_{2}$ are said to be quasi-isomorphic if there is a third chain complex $C_{0}$ and quasi-isomorphisms from $C_{0}$ to $C_{1}$ and $C_{0}$ to $C_{2}$. The following lemma is standard.

Lemma 2.1 Given chain maps $f: C_{1} \longrightarrow C_{2}$ and $f^{\prime}: C_{1}^{\prime} \longrightarrow C_{2}^{\prime}$ and quasi-isomorphisms $\phi_{1}: C_{1} \longrightarrow C_{1}^{\prime}$ and $\phi_{2}: C_{2} \longrightarrow C_{2}^{\prime}$ so that the composites $f^{\prime} \circ \phi_{1}$ is chain homotopic to $\phi_{2} \circ f$, there is an induced quasi-isomorphism $\Phi$ from $M(f)$ to $M\left(f^{\prime}\right)$ making the diagram

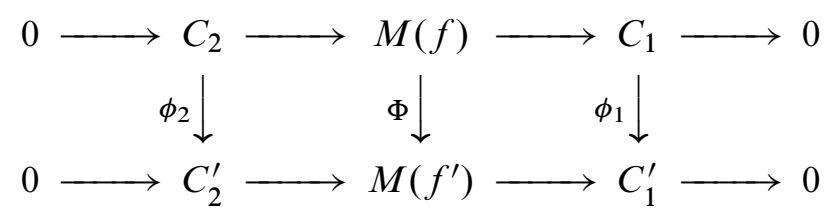

commutative. 
Proof Define $\Phi\left(a_{1} \oplus a_{2}\right)=\left(\phi_{1}\left(a_{1}\right),(-1)^{\operatorname{deg}\left(a_{1}\right)} H\left(a_{1}\right)+\phi_{2}\left(a_{2}\right)\right)$, where $H: C_{1} \longrightarrow$ $C_{2}^{\prime}$ is the homotopy between $f^{\prime} \circ \phi_{1}$ and $\phi_{2} \circ f$. It is easy to see that $\Phi$ is a chain map, and indeed that the labelled diagram is commutative. The map $\Phi$ is a quasiisomorphism by the five-lemma.

\subsection{Heegaard Floer complexes}

A pointed Heegaard diagram for a three-manifold $Y$ is a quadruple $(\Sigma, \boldsymbol{\alpha}, \boldsymbol{\beta}, z)$, where $\Sigma$ is an oriented surface of genus $g, \boldsymbol{\alpha}=\left\{\alpha_{1}, \ldots \alpha_{g}\right\}$ and $\boldsymbol{\beta}=\left\{\beta_{1}, \ldots, \beta_{g}\right\}$ are complete sets of attaching circles which specify $Y$, and

$$
z \in \Sigma-\alpha_{1}-\cdots-\alpha_{g}-\beta_{1}-\cdots-\beta_{g}
$$

is a reference point. It is explained in [16] that this data, together with some additional analytical choices (including a complex structure over $\Sigma$ ) leads to a collection chain complexes $C F^{-}(\Sigma, \boldsymbol{\alpha}, \boldsymbol{\beta}, z), C F^{\infty}(\Sigma, \boldsymbol{\alpha}, \boldsymbol{\beta}, z), C F^{+}(\Sigma, \boldsymbol{\alpha}, \boldsymbol{\beta}, z)$, and $\widehat{C F}(\Sigma, \boldsymbol{\alpha}, \boldsymbol{\beta}, z)$, which we refer to simply as $C F^{\circ}(\Sigma, \boldsymbol{\alpha}, \boldsymbol{\beta}, z)$. These complexes are constructed from a suitable variant of Lagrangian Floer homology in the $g$-fold symmetric product of $\Sigma$. Specifically, letting

$$
\mathbb{T}_{\alpha}=\alpha_{1} \times \cdots \times \alpha_{g} \subset \operatorname{Sym}^{g}(\Sigma) \quad \text { and } \quad \mathbb{T}_{\beta}=\beta_{1} \times \cdots \times \beta_{g} \subset \operatorname{Sym}^{g}(\Sigma),
$$

the complex $C F^{\infty}(\Sigma, \boldsymbol{\alpha}, \boldsymbol{\beta}, z)$ is freely generated over $\mathbb{Z}$ by generators $[\mathbf{x}, i] \in\left(\mathbb{T}_{\alpha} \cap\right.$ $\left.\mathbb{T}_{\beta}\right) \times \mathbb{Z}$, endowed with a differential

$$
\partial[\mathbf{x}, i]=\sum_{\mathbf{y} \in \mathbb{T}_{\alpha} \cap \mathbb{T}_{\beta}} \sum_{\left\{\phi \in \pi_{2}(\mathbf{x}, \mathbf{y}) \mid \mu(\phi)=1\right\}} \# \widehat{\mathcal{M}}(\phi)\left[\mathbf{y}, i-n_{z}(\phi)\right],
$$

where $\pi_{2}(\mathbf{x}, \mathbf{y})$ denotes the space of homotopy classes of Whitney disks from $\mathbf{x}$ to $\mathbf{y}, \mu(\phi)$ denotes its Maslov index, $\widehat{\mathcal{M}}(\phi)$ denotes the moduli space of pseudoholomorphic representatives of $\phi$ (with respect to some suitably generic perturbation), divided out by the natural translation action, and $n_{z}(\phi)$ denotes the algebraic intersection number of $\phi$ with the locus $\{z\} \times \operatorname{Sym}^{g-1}(\Sigma) . C F^{-}(\Sigma, \boldsymbol{\alpha}, \boldsymbol{\beta}, z)$ is the subcomplex generated by $[\mathbf{x}, i]$ with $i<0, C F^{+}(\Sigma, \boldsymbol{\alpha}, \boldsymbol{\beta}, z)$ is its quotient complex (ie generated by $i \geq 0)$, and $\widehat{C F}(\Sigma, \boldsymbol{\alpha}, \boldsymbol{\beta}, z)$ is the subcomplex of $C F^{+}$generated by pairs with $i=0$. These complexes are modules over the polynomial algebra $\mathbb{Z}[U]$, where $U \cdot[\mathbf{x}, i]=[\mathbf{x}, i-1]$. We have induced $\mathbb{Z}[U]$-actions on their homology groups $H F^{\infty}(Y), H F^{-}(Y), H F^{+}(Y)$, and $\widehat{H F}(Y)$ respectively.

The homology groups of these complexes are the Heegaard Floer homology groups of $Y$. As the notation suggests, although the chain complexes are defined using several choices (including a Heegaard diagram for $Y$ and a choice of complex structure over 
$\Sigma$ ), the homology groups on only the homeomorphism type of the underlying threemanifold. In fact, the proof of topological invariance from [16] actually proves more; it shows that that the chain homotopy type of the chain complexes $C F^{\circ}(\Sigma, \boldsymbol{\alpha}, \boldsymbol{\beta}, z)$ is a topological invariant of $Y$. As a shorthand, we let $C F^{\circ}(Y)$ denote the Heegaard Floer complex of $Y$ for some choice of Heegaard diagram (and auxilliary choices).

The Heegaard Floer complexes come with some additional structure. For example, there is a splitting of $C F^{+}(Y)$ into summands indexed by $\operatorname{Spin}^{c}$ structures over $Y$,

$$
C F^{+}(Y)=\bigoplus_{\mathfrak{t} \in \operatorname{Spin}^{c}(Y)} C F^{+}(Y, \mathfrak{t})
$$

These complexes are typically $\mathbb{Z} / 2 \mathbb{Z}$-graded, but when $Y$ is a rational homology three-sphere, or more generally, when $\mathfrak{t}$ is a $\operatorname{Spin}^{c}$ structure whose first Chern class is torsion, then $C F^{+}(Y, \mathfrak{t})$ is naturally a $\mathbb{Q}$-graded complex (cf Ozsváth-Szabó [20; 12]).

In a slight abuse of notation, we will in fact write $C F^{+}(Y)$ for a chain complex which is quasi-isomorphic to a chain complex for $Y$ with respect to some choice of Heegaard diagram. In the cases where $C F^{+}(Y)$ has extra structure (eg in the case $Y$ is a rational homology sphere and hence its Heegaard Floer complexes are $\mathbb{Q}$-graded), we require that our candidate have that additional structure, and the quasi-isomorphism preserves it.

It is shown in [20] that Heegaard Floer homology is natural under cobordisms. Specifically, in the present paper, three-manifolds $Y$ will always be closed and oriented. A cobordism $W$ from $Y_{1}$ to $Y_{2}$ is a smooth, connected, compact four-manifold with two boundary components $-Y_{1}$ and $Y_{2}$ (with respect to their boundary orientations). We sometimes write this as $W: Y_{1} \longrightarrow Y_{2}$. Of course, such a cobordism can be "turned around" and viewed as a cobordism $W:-Y_{2} \longrightarrow-Y_{1}$. Given a cobordism $W: Y_{1} \longrightarrow Y_{2}$, we say that two $\operatorname{Spin}^{c}$ structures $\mathfrak{t}_{i} \in \operatorname{Spin}^{c}\left(Y_{i}\right)$ for $i=1,2$ are $\operatorname{Spin}^{c}$ cobordant if there is a $\operatorname{Spin}^{c}$ structure $\mathfrak{s} \in \operatorname{Spin}^{c}(W)$ with $\mathfrak{s} \mid Y_{i}=\mathfrak{t}_{i}$ for $i=1,2$.

Suppose now that $W: Y_{1} \longrightarrow Y_{2}$ is a cobordism, equipped with a $\operatorname{Spin}^{c}$ structure $\mathfrak{s}$ whose restrictions $\mathfrak{t}_{i}=\left.\mathfrak{s}\right|_{Y_{i}}$ for $i=1,2$ both have torsion first Chern class, then there is an induced chain map

$$
f_{W, \mathfrak{s}}^{+}: C F^{+}\left(Y_{1}, \mathfrak{t}_{1}\right) \longrightarrow C F^{+}\left(Y_{2}, \mathfrak{t}_{2}\right)
$$

which is homogeneous of degree

$$
\frac{c_{1}(\mathfrak{s})^{2}-2 \chi(W)-3 \sigma(W)}{4}
$$

Algebraic 83 Geometric Topology, Volume 8 (2008) 
(cf [20, Theorem 7.1]). In fact, the $\mathbb{Q}$-grading on Floer homology is characterized by the above formula, and the normalization that $H F_{d}^{+}\left(S^{3}\right)$ is trivial for all $d<0$, non-trivial in degree $d=0$.

Sometimes, we will find it convenient to pass to a variant of Heegaard Floer homology parameterized by an integer $\delta \geq 0$ which interpolates between $\widehat{C F}$ and $C F^{+}$, which we write as $C F^{\delta}$. The generators of $C F^{\delta}$, now, are pairs $[\mathbf{x}, i]$ where $0 \leq i \leq \delta$, endowed with the induced differential from $C F^{+}$. In other words, $C F^{\delta}$ is the subcomplex of $C F^{+}(Y)$ which is the kernel of multiplication by $U^{\delta+1}$ (in particular, in the case where $\delta=0$, this construction gives $\widehat{C F}$ ).

\subsection{Integral surgeries on knots}

Let $K \subset Y$ be a null-homologous knot. Then, there is a canonical Seifert framing on $K$, giving rise to a curve $\lambda$ in the boundary of the tubular neighborhood of the knot $K, \operatorname{nd}(K)$, which meets the meridian $\mu$ in a single point. Given any integer $n$, the three-manifold $Y_{n}(K)$ denotes the new three-manifold obtained by $n$-Dehn filling on the complement $Y-\operatorname{nd}(K)$; ie this is a three-manifold obtained by filling $Y-\operatorname{nd}(K)$ with a solid torus whose new meridian is given by $n \cdot \mu+\lambda$. Of course, Dehn filling makes sense for arbitrary rational numbers, but we restrict attention here to integral surgeries. For such a surgery, in fact, there is also a canonical four-manifold $W_{n}(K)$ which is obtained by attaching a two-handle to $[0,1] \times Y$ with framing $n$ along $K$. This gives a cobordism from $Y$ to $Y_{n}(K)$. In our applications, we find it sometimes convenient to consider the cobordism $W_{n}^{\prime}(K): Y_{n}(K) \longrightarrow Y$ obtained by turning around the cobordism $-W_{n}(K):-Y \longrightarrow-Y_{n}(K)$.

Fix a Seifert surface $F$ for $K$, and let $\widehat{F} \subset W_{n}^{\prime}(K)$ denote the surface obtained by capping off $F$ in $W_{n}^{\prime}(K)$. Suppose that $\mathfrak{u}$ is a $\operatorname{Spin}^{c}$ structure over $Y_{n}(K)$ which admits an extension $\mathfrak{s}$ over $W_{n}^{\prime}(K)$ with the property that

$$
\left\langle c_{1}(\mathfrak{s}),[\widehat{F}]\right\rangle-n \equiv 2 i \quad(\bmod 2 n) .
$$

Lemma 2.2 The correspondence $\mathfrak{u} \mapsto i$ determined by the above formula induces a surjection $\operatorname{Spin}^{c}\left(Y_{n}(K)\right) \longrightarrow \mathbb{Z} / n \mathbb{Z}$. In the case where $Y$ is an integer homology three-sphere, the map is an isomorphism. More generally, if we fix a $\operatorname{Spin}^{c}$ structure $\mathfrak{t}$ over $Y$, the set of $\operatorname{Spin}^{c}$ structures over $Y_{n}(K)$ which are $\operatorname{Spin}^{c}$-cobordant to $\mathfrak{t}$ over $W_{n}^{\prime}(K)$ is identified with $\mathbb{Z} / n \mathbb{Z}$ under this correspondence.

Proof This is straightforward. 
Of course, in the case where $Y$ is an integral homology three-sphere, the above correspondence between $\operatorname{Spin}^{c}$ structures over $Y_{n}(K)$ and $\mathbb{Z} / n \mathbb{Z}$ depends on the Seifert surface only through its induced orientation on $K$. Moreover, if we fix $i \in \mathbb{Z} / n \mathbb{Z}$ and use the opposite orientation on $K$, the induced $\operatorname{Spin}^{c}$ structure over $Y_{n}(K)$ is conjugated.

For $i \in \mathbb{Z} / n \mathbb{Z}$, when $Y$ is an integral homology three-sphere we write $H^{+}\left(Y_{n}(K), i\right)$ for the Heegaard Floer homology of $Y_{n}(K)$ calculated in the $\operatorname{Spin}^{c}$ structure corresponding to $i \in \mathbb{Z} / n \mathbb{Z}$ under the correspondence from the above lemma.

For $n \neq 0$, the lens space $L(n, 1)$ can be viewed as $n$-surgery on the unknot. For $n>0$, let

$$
d(n, i)=-\max _{\{s \in \mathbb{Z} \mid s \equiv i(\bmod n)\}} \frac{1}{4}\left(1-\frac{(n+2 s)^{2}}{n}\right)
$$

and let $d(-n, i)=-d(n, i)$. It can be shown that $d(n, i)$ is the smallest degree in which $\mathrm{HF}^{+}(L(n, 1), i)$ is non-trivial (cf Ozsváth-Szabó [12, Proposition 4.8]).

\subsection{Knot Floer homology and large $n$ surgeries}

We follow here the notation on knot Floer homology from [14]. A knot $K \subset Y$ has a compatible Heegaard diagram $(\Sigma, \boldsymbol{\alpha}, \boldsymbol{\beta}, w, z)$, where $(\Sigma, \boldsymbol{\alpha}, \boldsymbol{\beta})$ is a Heegaard diagram for $Y$, the knot $K$ is supported in the handlebody specified by $\boldsymbol{\beta}$, where it is a standard unknotted circle which is dual to the $\beta_{g}$-attaching disk, and $w$ and $z$ are a pair of reference points close to, and lying on either side of $\beta_{g}$.

This gives rise to a map

$$
\underline{\mathfrak{s}}: \mathbb{T}_{\alpha} \cap \mathbb{T}_{\beta} \longrightarrow \mathbb{Z}
$$

which is half of the first Chern class of the relative $\operatorname{Spin}^{c}$ structure belonging to $\mathbf{x} \in \mathbb{T}_{\alpha} \cap \mathbb{T}_{\beta}$ evaluated on a Seifert surface for $K$. The knot chain complex $C$ described in the introduction is then generated by $[\mathbf{x}, i, j] \in\left(\mathbb{T}_{\alpha} \cap \mathbb{T}_{\beta}\right) \times \mathbb{Z} \times \mathbb{Z}$, satisfying $\underline{\mathfrak{s}}(\mathbf{x})+(i-j)=0$, endowed with the differential

$$
\partial[\mathbf{x}, i, j]=\sum_{\mathbf{y} \in \mathbb{T}_{\alpha} \cap \mathbb{T}_{\beta}} \sum_{\left\{\phi \in \pi_{2}(\mathbf{x}, \mathbf{y}) \mid \mu(\phi)=1\right\}} \# \widehat{\mathcal{M}}(\phi) \cdot\left[\mathbf{y}, i-n_{w}(\phi), j-n_{z}(\phi)\right] .
$$

This complex is given the filtration function $\mathcal{F}[\mathbf{x}, i, j]=(i, j)$. The forgetful map $[\mathbf{x}, i, j] \longrightarrow[\mathbf{x}, i]$ induces an isomorphism between $C$ and $C F^{\infty}(Y)$, sending $C\{i \geq 0\}$ isomorphically to $C F^{+}(Y)$. 
The following result is proved in [14, Theorem 4.4], but we sketch the proof again for the reader's convenience. (In fact, a corresponding statement holds for a null-homologous knot in an arbitrary closed, oriented three-manifold.)

Theorem 2.3 Let $K \subset Y$ be a null-homologous knot in an integral homology threesphere. There is an integer $N$ with the property that for all $m \geq N$ and all $t \in \mathbb{Z} / m \mathbb{Z}$, $C F^{+}\left(Y_{m}(K), t\right)$ is represented by the chain complex $A_{s}^{+}=C\{\min (i, j-s) \geq 0\}$ where $s \equiv t(\bmod m)$ and $|s| \leq m / 2$, in the sense that there are isomorphisms (of relatively $\mathbb{Z}$-graded $\mathbb{Z}[U]$-complexes)

$$
\Psi_{m, s}^{+}: C F^{+}\left(Y_{m}(K), t\right) \longrightarrow A_{s}^{+} .
$$

Moreover, if $\mathfrak{x}_{s}$ and $\mathfrak{y}_{s}$ denote the $\operatorname{Spin}^{c}$ structures over $W_{m}^{\prime}(K)$ with

$$
\left\langle c_{1}\left(\mathfrak{x}_{s}\right),[\widehat{F}]\right\rangle+m=2 \operatorname{sresp} .\left\langle c_{1}\left(\mathfrak{y}_{s}\right),[\widehat{F}]\right\rangle-m=2 s,
$$

then $v_{s}^{+}$and $h_{s}^{+}$correspond to the maps induced by the cobordism $W_{m}^{\prime}(K)$ endowed with the $\mathfrak{x}_{s}$ and $\mathfrak{y}_{s}$ respectively, in the sense that the following squares commute:

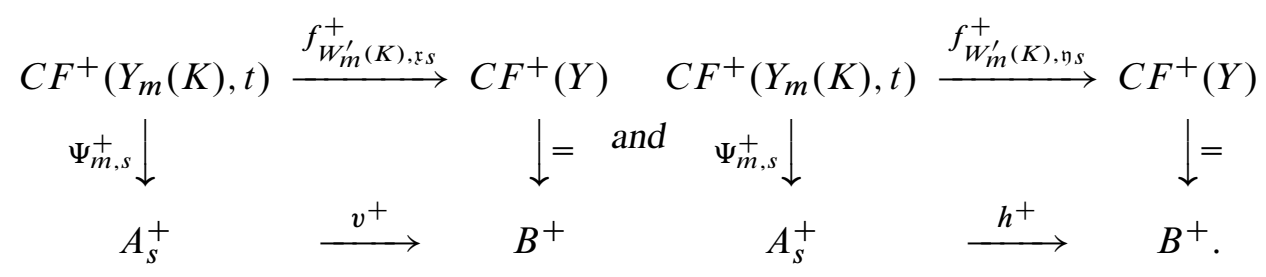

Sketch of proof Let $(\Sigma, \boldsymbol{\alpha}, \boldsymbol{\gamma}, \boldsymbol{\beta}, w, z)$ be a Heegaard diagram for the cobordism $W_{m}^{\prime}: Y_{m}(K) \longrightarrow Y$, containing the pair of basepoints $w$ and $z$ one on each side of the meridian for $Y$. In particular, here the three-manifold $Y_{\alpha, \gamma} \cong Y_{m}(K), Y_{\gamma, \beta} \cong$ $\#^{g-1}\left(S^{2} \times S^{1}\right), Y_{\alpha, \beta} \cong Y$.

For $s \in \mathbb{Z}$, we define the map $\Psi_{m, s}^{+}: C F^{+}\left(Y_{m}(K),[s]\right) \longrightarrow C\{\max (i, j-s) \geq 0\}$ by $\Psi_{m, s}^{+}[\mathbf{x}, i]=\sum_{\mathbf{y} \in \mathbb{T}_{\alpha} \cap \mathbb{T}_{\beta}} \sum_{\left\{\psi \in \pi_{2}(\mathbf{x}, \Theta, \mathbf{y}) \mid n_{w}(\psi)-n_{z}(\psi)=s-\underline{\mathfrak{s}}(\mathbf{x})\right\}} \# \mathcal{M}(\psi)\left[\mathbf{y}, i-n_{w}(\psi), i-n_{z}(\psi)\right]$,

where here $\Theta \in H F^{\leq 0}\left(Y_{\beta, \gamma}\right)$ is a generator. The constraint on homotopy classes of triangles is equivalent to the constraint that the first Chern class $\mathfrak{s}$ induced by the homotopy class $\psi \in \pi_{2}(\mathbf{x}, \Theta, \mathbf{y})$ and the basepoint $w$ satisfies

$$
\left\langle c_{1}(\mathfrak{s}),[\widehat{F}]\right\rangle+m=2 s .
$$

It is shown in the proof of [14, Theorem 4.4] for all sufficiently large $m$ and all $t \in \mathbb{Z} / m \mathbb{Z}$, we have that $\Psi_{m, s}^{+}$induces an isomorphism of chain complexes from 
$C F^{+}\left(Y_{m}(K), t\right)$ to $C\{\max (i, j-s) \geq 0\}$ for $|s| \leq \frac{m}{2}$ and $s \equiv t(\bmod m)$. Postcomposing $\Psi_{m, s}^{+}$with the projection $C\{\max (i, j-s) \geq 0\}$ to $C\{i \geq 0\}$ (ie $v^{+}$), we obtain the map induced by the cobordism $W_{m}^{\prime}(K)$ equipped with the $\operatorname{Spin}^{c}$ structure $\mathfrak{x}_{s}$; ie the square in the statement of the theorem on the left commutes.

For commutativity of the second square, observe that the composite $h^{+} \circ \Psi_{m, s}^{+}$is identified with a map $C F^{+}\left(Y_{m}(K)\right)$ to $C F^{+}(Y)$ induced by counting pseudo-holomorphic triangles in a fixed $\operatorname{Spin}^{c}$ equivalence class, using the reference point $z$ (rather than $w)$. As we have seen, with respect to the reference point $w$, this $\operatorname{Spin}^{c}$ equivalence class induces the $\operatorname{Spin}^{c}$ structure $\mathfrak{x}_{s}$; thus, with respect to the reference point $z$, the induced $\operatorname{Spin}^{c}$ structure is $\mathfrak{y}_{s}=\mathfrak{x}_{s}-\operatorname{PD}[\widehat{F}]$.

It follows from the above statement that there are corresponding identifications between $\widehat{C F}\left(Y_{m}(K), i\right)$ with $C\{\max (i, j-s)=0\}$, as well.

Corollary 2.4 Let $K \subset Y$ be a null-homologous knot in an integer homology threesphere, and fix an integer $\delta \geq 0$. There are constants $C_{1}$ and $C_{2}$ (depending on the knot $K$ and the choice of $\delta \geq 0$ ) with the property that for all sufficiently large $N$, and any choice of $i$, there is a chain complex $C F^{\delta}\left(Y_{N}, i\right)$ such that

$$
\text { (4) }\left|\max \operatorname{gr} C F^{\delta}\left(Y_{N}, i\right)-\min \operatorname{gr} C F^{\delta}(Y)+\max _{s \equiv i}(\bmod N) \frac{1}{4}\left(1-\frac{|2 s+N|^{2}}{N}\right)\right| \leq C_{2} \text {. }
$$

Proof Both statements follow from Theorem 2.3. The first statement is an immediate consequence of the homogeneous identification for all sufficiently large $N$ of $C F^{+}\left(Y_{N}(K), i\right)$ with $A_{i}^{+}$(which in turn is independent of $N$ ). For the second assertion, observe that either $\mathfrak{x}_{i}$ or $\mathfrak{y}_{i}$ is the $\operatorname{Spin}^{c}$ structure over $W_{N}^{\prime}(K)$ for which $c_{1}(\mathfrak{s})^{2}$ is maximized, amongst all $\mathrm{Spin}^{c}$ structures whose restriction to $Y_{N}(K)$ corresponds to $i$. (In fact, it is $\mathfrak{x}_{i}$ if $i \geq 0$, and $\mathfrak{y}_{i}$ if $i \leq 0$.) Moreover, the induced map on $W_{N}^{\prime}(K)$ is realized as $v^{+}$or $h^{+}$, according to whether or not $i \geq 0$. It is now immediate to see that for $i \geq 0$ resp $i \leq 0$, there is a constant $C_{2}$ with the property that all of $C F^{+}(Y)$ is contained within $C_{2}$ grading levels of the image of $v^{+}$resp. $h^{+}$. The second inequality follows from this observation, together with the is a shift in the gradings in (1) specialized to the cobordism $W_{N}^{\prime}(K)$.

\subsection{An example: the unknot}

In this subsection, we consider a special case of Theorem 1.1: the case of the unknot $K$ in the three-sphere. 
For the unknot $K$, the knot Floer complex $C F K^{\infty}(K)$ is generated over $\mathbb{Z}$ by a sequence of generators $\left\{x_{i}\right\}_{i \in \mathbb{Z}}$ with $\mathcal{F}\left(x_{i}\right)=(i, i)$, and $U\left(x_{i}\right)=x_{i-1}$.

Let $\mathcal{T}^{+}$denote the $\mathbb{Z}[U]$ module $\mathbb{Z}\left[U, U^{-1}\right] / U \cdot \mathbb{Z}[U]$.

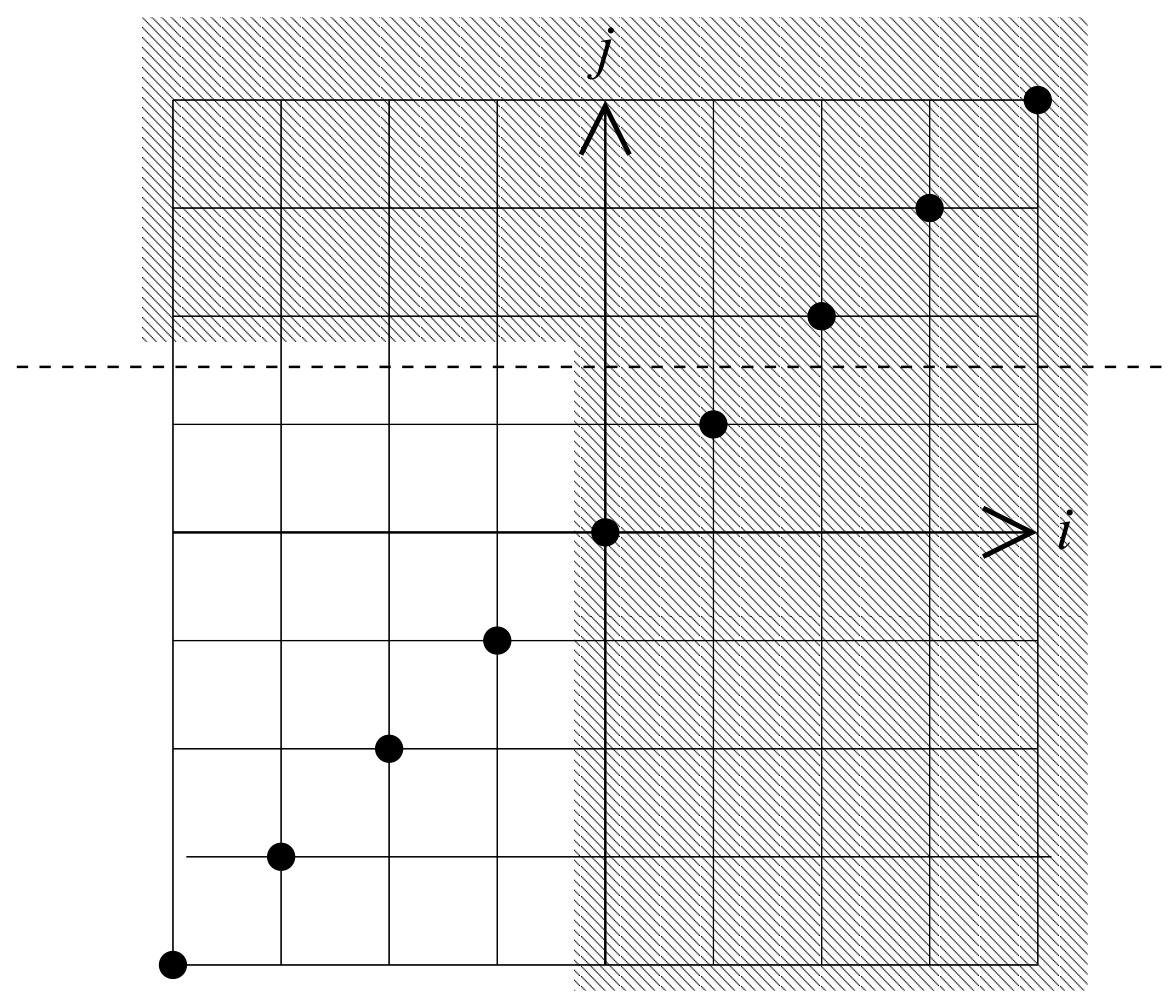

Figure 1: The doubly-filtered knot complex for the unknot. We have illustrated the doubly-filtered complex for the unknot: each dot at the location $(i, j)$ denotes a $\mathbb{Z}$-generator for the complex $C$ whose filtration level is $(i, j)$ (and indeed, they are all located at $(i, i))$. The hatched region represents $A_{2}^{+}$, while projection to the region above the dotted line represents $h_{2}^{+}$. The two generators in $A_{2}^{+}$but not above the dotted line represent the two kernel elements of $h_{2}^{+}$.

For the unknot, we have that $A_{s}^{+}=\mathcal{T}^{+}$for all $s$, and also $B_{s}^{+}=\mathcal{T}^{+}$. Moreover, the maps

$$
v_{s}^{+}: A_{s}^{+} \longrightarrow B_{s}^{+} \text {and } h_{s}^{+}: A_{s}^{+} \longrightarrow B_{s+n}^{+}
$$

Algebraic 83 Geometric Topology, Volume 8 (2008) 
can be explicitly identified (up to multiplication by \pm 1 ) as endomorphisms of $\mathcal{T}^{+}$:

$$
\begin{gathered}
v_{s}^{+}= \begin{cases}1 & \text { if } s \geq 0 \\
U^{-s} & \text { if } s \leq 0\end{cases} \\
h_{s}^{+}= \begin{cases}U^{s} & \text { if } s \geq 0 \\
1 & \text { if } s \leq 0 .\end{cases}
\end{gathered}
$$

For fixed $i \in \mathbb{Z} / n \mathbb{Z}$, let $\mathbb{A}_{i}^{+}=\bigoplus_{s \equiv i(\bmod n)} A_{s}^{+}$and $\mathbb{B}_{i}^{+}=\bigoplus_{s \equiv i(\bmod n)} B_{s}^{+}$, and

$$
D_{n, i}^{+}: \mathbb{A}_{i}^{+} \longrightarrow \mathbb{B}_{i}^{+}
$$

denote the map obtained by restricting $D_{n}^{+}$. Clearly, the mapping cone of $D_{n}^{+}$splits as a direct sum of the mapping cones $D_{n, i}^{+}$over all $i \in \mathbb{Z} / n \mathbb{Z}$.

It is easy to see that the homology of the mapping cone

$$
D_{n, i}^{+}: \mathbb{A}^{+} \longrightarrow \mathbb{B}^{+}
$$

is isomorphic to $\mathcal{T}^{+}$. This, of course, is consistent with Theorem 1.1, together with the fact that the Floer homology of the lens space $L(n, 1)$ (which in turn is $n$ surgery on the unknot) has $H^{+}(L(n, 1), i) \cong \mathcal{T}^{+}$for each $i \in \mathbb{Z} / n \mathbb{Z}$ (cf [16, Proposition 8.1]). More explicitly, in the case where $n>0$, it is easy to see that $D_{n, i}^{+}$is surjective, and its kernel is identified with the image of an injection

$$
\iota: \mathcal{T}^{+} \longrightarrow \mathbb{A}^{+}
$$

given by the formula

$$
\iota(\xi)=\left\{U^{\epsilon(\sigma+k n, n)} \cdot \xi\right\}_{k \in \mathbb{Z}},
$$

where here $\sigma$ is the representative for $i(\bmod n)$ with $0 \leq \sigma<n$, and

$$
\epsilon(\sigma+k n, n)= \begin{cases}k \sigma+\left(\frac{k(k-1) n}{2}\right) & \text { if } k \geq 0 \\ (k+1) \sigma+\left(\frac{k(k+1) n}{2}\right) & \text { if } k<0 .\end{cases}
$$

Indeed, $\iota$ induces the isomorphism of $\mathcal{T}^{+}$with $H_{*}\left(M\left(D_{n, i}^{+}: \mathbb{A}^{+} \longrightarrow \mathbb{B}^{+}\right)\right)$.

Dually, if $n>0$, then $D_{-n, i}^{+}$is injective, and its cokernel is isomorphic to $\mathcal{T}^{+}$. In fact, for any $\delta \geq 0$, let $\mathbb{A}^{\delta}$ resp. $\mathbb{B}^{\delta}$ denote the kernel of $U^{\delta+1}$ on $\mathbb{A}^{+}$resp $\mathbb{B}^{+}$, and let

$$
D_{-n, i}^{\delta}: \mathbb{A}^{\delta} \longrightarrow \mathbb{B}^{\delta}
$$

denote the restriction of $D_{-n, i}^{+}$. There is a map

$$
\pi: \mathbb{B}^{\delta} \longrightarrow \mathbb{Z}[U] / U^{\delta+1}
$$

Algebraic $8 \mathcal{G}$ Geometric Topology, Volume 8 (2008) 
defined by

$$
\pi\left(\left\{\eta_{\sigma+k n}\right\}_{k \in \mathbb{Z}}\right)=\sum_{k \in \mathbb{Z}}(-1)^{k} U^{\epsilon(-(\sigma+k n), n)} \eta_{\sigma+k n}
$$

which vanishes on the image of $D_{-n, i}^{\delta}\left(A^{\delta}\right)$, and inducing an isomorphism

$$
H_{*}\left(M\left(D_{-n, i}^{\delta}: \mathbb{A}^{\delta} \longrightarrow \mathbb{B}^{\delta}\right) \cong \mathbb{Z}[U] / U^{\delta+1} .\right.
$$

\subsection{Algebra}

Consider the polynomial algebra $\mathbb{Z}[U]$ in a single variable. A graded $\mathbb{Z}[U]$ module is a $\mathbb{Q}$-graded module $M$ over the ring $\mathbb{Z}[U]$ with the property that the endomorphism $U: M \longrightarrow M$ is homogeneous with degree -2 . For $d \in \mathbb{Z}$, let $M_{d}$ denote the the subgroup generated by homogeneous elements of degree $d$, and let $M_{\leq k}$ denote the sum

$$
M_{\leq k}=\bigoplus_{\{d \leq k\}} M_{d}
$$

Note that for all integers $k, M_{\leq k}$ is a $\mathbb{Z}[U]$-submodule of $M$.

We say that a graded $\mathbb{Z}[U]$-module is of $\mathrm{HF}^{+}$type if for all sufficiently large degrees $k$, the endomorphism

$$
U: M_{d} \longrightarrow M_{d-2}
$$

is an isomorphism, and also for all sufficiently small degrees, $M_{d}=0$. Of course, if $Y$ is any three-manifold and $\mathfrak{t} \in \operatorname{Spin}^{c}(Y)$ is a $\operatorname{Spin}^{c}$ structure whose first Chern class is torsion, then $\mathrm{HF}^{+}(Y, \mathfrak{t})$ is a $\mathbb{Z}[U]$-module of $H F^{+}$-type.

The following lemma is straightforward.

Lemma 2.5 Let $A$ and $B$ be a pair of graded $\mathbb{Z}[U]$-modules of $\mathrm{HF}^{+}$-type. For any integer $c$ there is a constant $D$ with the property that for all $k \geq D$, any homogeneous map of degree $c$ defined on $A_{\leq k}$

$$
f_{\leq k}: A_{\leq k} \longrightarrow B_{\leq c+k}
$$

can be uniquely extended to a $\mathbb{Z}[U]$-module map

$$
f: A \longrightarrow B \text {. }
$$

In particular, if $A$ and $B$ are of $H F^{+}$-type then there is a $\delta \geq 0$ with the property that if $A_{\leq \delta} \cong B_{\leq \delta}$, then $A \cong B$. 
Proof Since $A$ and $B$ are of $H F^{+}$type, for all sufficiently large $\ell$ and all nonnegative integers $m$, the maps

$$
U^{m}: A_{\ell+2 m} \longrightarrow A_{\ell}
$$

and

$$
U^{m}: B_{\ell+2 m+c} \longrightarrow B_{\ell+c}
$$

are isomorphisms. Choose then $k \geq \ell+2$, and define

$$
f(a)= \begin{cases}f_{\leq k}(a) & \text { if } \operatorname{gr}(a) \leq k \\ U^{-m} f_{\leq k}\left(U^{m}(a)\right) & \text { where } m \text { is chosen so that } \ell \leq \operatorname{gr}(a)-2 m \leq \ell+2 .\end{cases}
$$

It is easy to see that $f$ is a canonically-defined extension of $f_{\leq k}$. The last claim follows immediately.

Definition 2.6 A chain complex $C$ over the ring $\mathbb{Z}[U]$ is said to be of $C F^{+}$-type if it is quasi-isomorphic (over $\mathbb{Z}[U]$ ) to a chain complex of the form $C^{\prime} \otimes_{\mathbb{Z}[U]}$ $\mathbb{Z}\left[U, U^{-1}\right] / \mathbb{Z}[U]$, where $C^{\prime}$ is a finitely generated, free chain complex over $\mathbb{Z}[U]$.

For example, the chain complex $C F^{+}(Y)$ is of $C F^{+}$-type (the chain complex $C F^{-}(Y)$ plays the role here of $C^{\prime}$ ). It is also straightforward to see that if $C$ is a graded $\mathbb{Z}[U]-$ complex which is of $\mathrm{CF}^{+}$-type, then its homology is of $\mathrm{HF}^{+}$-type. The mapping cones $\mathbb{X}^{+}(n)$ from the introduction can also be seen to be of $C F^{+}$-type (cf Lemma 4.3 below).

Let $C$ be a chain complex over $\mathbb{Z}[U]$, and fix an integer $\delta \geq 0$. Let $C^{\delta}$ denote the subcomplex of elements in the kernel of multiplication by $U^{\delta+1}$.

Lemma 2.7 Let $A$ and $B$ be two chain complexes of graded $\mathbb{Z}[U]$-modules which are of $\mathrm{CF}^{+}$-type. For any $c$, there is a constant $D$ with the property that for all integers $\delta \geq D$, any homogeneous map of degree $c$ defined on

$$
f^{\delta}: H_{*}\left(A^{\delta}\right) \longrightarrow H_{*}\left(B^{\delta}\right)
$$

can be uniquely extended to a $\mathbb{Z}[U]$-module map

$$
f: H_{*}(A) \longrightarrow H_{*}(B) .
$$

In particular, if $A$ and $B$ are graded $\mathbb{Z}[U]$-complexes which are of $C F^{+}$-type, then there is an integer $\delta \geq 0$ with the property that if $H_{*}\left(A^{\delta}\right) \cong H_{*}\left(B^{\delta}\right)$, then $H_{*}(A) \cong$ $H_{*}(B)$. 
Proof Consider the short exact sequence

$$
0 \longrightarrow A^{\delta} \longrightarrow A \stackrel{U^{\delta+1}}{\longrightarrow} A \longrightarrow 0 .
$$

note that the connecting homomorphism is a homogeneous map of degree $2 \delta+1$. In particular, for any $k$, we can choose $\delta$ so that $H_{*}\left(A_{\leq k-2 \delta-1}\right)=0$, the induced long exact sequence gives an isomorphism $H_{*}\left(A_{\leq k}^{\delta}\right) \cong H_{*}\left(A_{\leq k}\right)$. The lemma then follows at once from Lemma 2.5 .

\section{An exact sequence for surgeries}

Theorem 1.1 hinges on the following exact sequence relating different surgeries on a knot in a three-manifold $Y$. Loosely speaking, the exact sequence is induced by the homology between the curve $(m+n, 1)$ in the torus with the sum of curves $(n, 1)$ and $(m, 0)$ (where here $m, n$, are integers and the homology classes of curves in the torus are written as $\left.(a, b) \in \mathbb{Z} \oplus \mathbb{Z} \cong H_{1}\left(T^{2} ; \mathbb{Z}\right)\right)$.

Theorem 3.1 Let $Y$ be a closed, oriented three-manifold, equipped with a nullhomologous knot $K$. Fix an integer $n$ and a positive integer $m$. Then, there is a long exact sequence

$$
\cdots \longrightarrow H F^{+}\left(Y_{n}(K)\right) \longrightarrow H F^{+}\left(Y_{m+n}(K)\right) \longrightarrow \bigoplus^{m} H F^{+}(Y) \longrightarrow \cdots
$$

and also a corresponding exact sequence using $\widehat{H F}$ in place of $\mathrm{HF}^{+}$. Indeed, there are $\mathbb{Z}[U]$-equivariant chain maps

$$
\begin{gathered}
f_{1}^{+}: C F^{+}\left(Y_{n}(K)\right) \longrightarrow C F^{+}\left(Y_{m+n}(K)\right) \\
f_{2}^{+}: C F^{+}\left(Y_{m+n}(K)\right) \longrightarrow \bigoplus^{m} C F^{+}(Y) \\
f_{3}^{+}: \bigoplus^{m} C F^{+}(Y) \longrightarrow C F^{+}\left(Y_{n}(K)\right)
\end{gathered}
$$

inducing the maps in the long exact sequence, and $\mathbb{Z}[U]$-equivariant quasi-isomorphisms

$$
\begin{aligned}
\phi^{+}: C F^{+}\left(Y_{n}(K)\right) & \longrightarrow M\left(f_{2}^{+}\right) \\
\psi^{+}: M\left(f_{2}^{+}\right) & \longrightarrow C F^{+}\left(Y_{n}(K)\right) .
\end{aligned}
$$

(See (8), (9), (11), (13), and (14) for the definitions of $f_{1}^{+}, f_{2}^{+}, f_{3}^{+}, \phi^{+}$, and $\psi^{+}$ respectively.) 
The proof follows very closely along the lines of various other previously-established exact sequences for surgeries. In particular, we assume familiarity with [15, Section $10]$, and continue in the notation set up there.

Proof of Theorem 3.1 Consider a pointed Heegaard diagram for $Y,(\Sigma, \boldsymbol{\alpha}, \boldsymbol{\beta}, z)$, with the property that $K$ is contained entirely inside the handlebody $U_{\beta}$, so that it is disjoint from the attaching disks bounding $\beta_{1}, \ldots, \beta_{g-1}$, and with the additional property that $\beta_{g}$ is a meridian for the knot. Let $\gamma_{g}$ be a simple, closed curve in $\Sigma$ disjoint from the $\beta_{1}, \ldots, \beta_{g-1}$ which specifies the $n$-framing of $K$. (In particular, if $\lambda_{g}$ is the canonical 0 -framing, then $\gamma_{g}$ is a smooth curve which is homologous to $n \cdot \beta_{g}+\lambda_{g}$.) We complete this to a $g$-tuple of attaching circles $\gamma$ by taking curves $\gamma_{1}, \ldots, \gamma_{g-1}$ which are small Hamiltonian translates of $\beta_{1}, \ldots, \beta_{g-1}$ respectively. Similarly, define $\boldsymbol{\delta}$, only this time $\delta_{g}$ corresponds to the framing $m+n$. Thus, $(\Sigma, \boldsymbol{\alpha}, \boldsymbol{\gamma})$ and $(\Sigma, \boldsymbol{\alpha}, \boldsymbol{\delta})$ are Heegaard diagrams for $Y_{n}(K)$ and $Y_{m+n}(K)$ respectively.

We place a basepoint $p$ on $\beta_{g}$, and consider twisted homology with coefficients in $\mathbb{Z} / m \mathbb{Z}$; ie write $\mathbb{Z}[\mathbb{Z} / m \mathbb{Z}]=\mathbb{Z}[T] /\left(T^{m}-1\right)$, and consider the chain complex $C F^{+}(Y) \otimes_{\mathbb{Z}} \mathbb{Z}[\mathbb{Z} / m \mathbb{Z}]$ endowed with the differential

$$
\underline{\partial}^{+}[\mathbf{x}, i]=\sum_{\mathbf{y} \in \mathbb{T}_{\alpha} \cap \mathbb{T}_{\beta}} \sum_{\left\{\phi \in \pi_{2}(\mathbf{x}, \mathbf{y}) \mid \mu(\phi)=1\right\}} \#\left(\frac{\mathcal{M}(\phi)}{\mathbb{R}}\right) \cdot T^{m_{p}(\phi)} \cdot\left[\mathbf{y}, i-n_{z}(\phi)\right]
$$

where as usual here $\mathbf{x} \in \mathbb{T}_{\alpha} \cap \mathbb{T}_{\beta}, i \geq 0, \pi_{2}(\mathbf{x}, \mathbf{y})$ denotes the space of homotopy classes of Whitney disks connecting $\mathbf{x}$ and $\mathbf{y}, \mu(\phi)$ denotes the Maslov index of $\phi$, and terms in the above equation for which $i-n_{z}(\phi)<0$ are to be dropped. Moreover, $m_{p}(\phi)$ denotes the multiplicity of the basepoint $p$ in the boundary of $\phi$; ie $p$ determines a codimension one submanifold $\beta_{1} \times \cdots \times \beta_{g-1} \times\{p\} \subset \mathbb{T}_{\beta}$, and we look at the intersection number with the restriction of the boundary of $\phi$ with this subset. We denote the complex by $C F^{+}(Y ; \mathbb{Z}[\mathbb{Z} / m \mathbb{Z}])$ (In the terminology of [16], this is the chain complex for $Y$ with twisted coefficients in $\mathbb{Z}[\mathbb{Z} / m \mathbb{Z}]$, where it is denoted $C F^{+}(Y ; \mathbb{Z}[\mathbb{Z} / m \mathbb{Z}])$, however, we drop the underline here in the interest of notational simplicity.) There is an isomorphism of chain complexes of modules over $\mathbb{Z}[\mathbb{Z} / m \mathbb{Z}]$,

$$
\theta: C F^{+}(Y ; \mathbb{Z}[\mathbb{Z} / m \mathbb{Z}]) \stackrel{\cong}{\longrightarrow} C F^{+}(Y) \otimes_{\mathbb{Z}} \mathbb{Z}[\mathbb{Z} / m \mathbb{Z}],
$$

where here the right-hand-side is endowed with the differential which is the original differential on $C F^{+}(Y)$ tensored with the identity map on $\mathbb{Z}[\mathbb{Z} / m \mathbb{Z}]$. This map is induced by fixing an intersection point $\mathbf{x}_{0} \in \mathbb{T}_{\alpha} \cap \mathbb{T}_{\beta}$, and defining

$$
\theta[\mathbf{x}, i]=T^{m_{p}(\phi)}[\mathbf{x}, i],
$$


where here $\phi \in \pi_{2}\left(\mathbf{x}, \mathbf{x}_{0}\right)$. Note that $m_{p}(\phi)$ depends on $\phi$ only through the choice of $\mathbf{x}_{0}$ and $\mathbf{x}$ (and indeed the map $\theta$ depends on the choice of $\mathbf{x}_{0}$ and the placement of $p$ through an overall multiple of a power of $T$ ). There is a corresponding identification

$$
H F^{+}(Y ; \mathbb{Z}[\mathbb{Z} / m \mathbb{Z}]) \cong H F^{+}(Y) \otimes_{\mathbb{Z}} \mathbb{Z}[\mathbb{Z} / m \mathbb{Z}] \cong \bigoplus^{m} H F^{+}(Y)
$$

First, we define the map $f_{1}^{+}$. The map $f_{1}^{+}$is defined by counting pseudo-holomorphic triangles between $\mathbb{T}_{\alpha}, \mathbb{T}_{\gamma}$, and $\mathbb{T}_{\delta}$. More precisely, note that the Heegaard triple $(\Sigma, \boldsymbol{\alpha}, \boldsymbol{\gamma}, \boldsymbol{\delta}, z)$ determines a four-manifold $X_{\alpha, \gamma, \delta}$ with three boundary components

$$
Y_{\alpha, \gamma} \cong Y_{n}(K), \quad Y_{\alpha, \delta} \cong Y_{n+m}(K), \text { and } Y_{\gamma, \delta} \cong \#^{g-1}\left(S^{2} \times S^{1}\right) \# L(m, 1) .
$$

We will fix a $\operatorname{Spin}^{c}$ structure over $Y_{\gamma, \delta}$ by the following convention.

Definition 3.2 The lens space $L(m, 1)$ bounds a tubular neighborhood of a sphere $S$ whose self-intersection number is $m$. The canonical $\operatorname{Spin}^{c}$ structure $\ell \in$ $\operatorname{Spin}^{c}(L(m, 1))$ is the one which bounds a $\operatorname{Spin}^{c}$ structure $\mathfrak{s}$ over the tubular neighborhood which satisfies $\left\langle c_{1}(\mathfrak{s}),[S]\right\rangle=m$. For the connected sum $L(m, 1) \#\left(\#^{g-1}\left(S^{2} \times\right.\right.$ $\left.S^{1}\right)$ ), the canonical $\operatorname{Spin}^{c}$ structure is the one whose first Chern class is torsion and whose restriction to $L(m, 1)$ is the canonical $\operatorname{Spin}^{c}$ structure.

Let $\Theta_{\gamma \delta}$ denote the Floer homology class corresponding to the generator (over $\left.\Lambda^{*} H_{1}\left(Y_{\gamma, \delta}\right) \otimes \mathbb{Z}[U]\right)$ of

$$
H F^{\leq 0}\left(Y_{\gamma, \delta}, \ell\right) \cong \Lambda^{*} H^{1}\left(Y_{\gamma, \delta}\right) \otimes \mathbb{Z}[U]
$$

in its canonical $\operatorname{Spin}^{c}$ structure $\ell$. For simplicity, we can arrange for the homology class $\Theta_{\gamma \delta}$ to be represented by a single intersection point in $\mathbb{T}_{\gamma} \cap \mathbb{T}_{\delta}$, which we also denote by $\Theta_{\gamma \delta}$.

We then define

$$
f_{1}^{+}([\mathbf{x}, i])=\sum_{\mathbf{y} \in \mathbb{T}_{\alpha} \cap \mathbb{T}_{\delta}} \sum_{\left\{\psi \in \pi_{2}\left(\mathbf{x}, \Theta_{\gamma \delta}, \mathbf{y}\right) \mid \mu(\psi)=0\right\}} \# \mathcal{M}(\psi) \cdot\left[\mathbf{y}, i-n_{z}(\psi)\right] .
$$

Similarly, we define $f_{2}^{+}: C F^{+}\left(Y_{m+n}(K)\right) \longrightarrow C F^{+}(Y ; \mathbb{Z}[\mathbb{Z} / m \mathbb{Z}])$ by

$$
\text { (9) } f_{2}^{+}([\mathbf{y}, i])=\sum_{\mathbf{w} \in \mathbb{T}_{\alpha} \cap \mathbb{T}_{\beta}} \sum_{\left\{\psi \in \pi_{2}\left(\mathbf{y}, \Theta_{\delta \beta}, \mathbf{w}\right) \mid \mu(\psi)=0\right\}} \# \mathcal{M}(\psi) \cdot\left[\mathbf{w}, i-n_{z}(\psi)\right] \cdot T^{m_{p}(\psi)} \text {. }
$$

The map $f_{2}^{+}$can be thought of more invariantly as a weighted sum of maps induced by cobordisms, as follows. For our Heegaard triple $(\Sigma, \boldsymbol{\alpha}, \boldsymbol{\delta}, \boldsymbol{\beta}, z)$, the doubly-periodic 
domains all have multiplicity zero at $p$, while the generator of the space of triplyperiodic domains modulo doubly-periodic ones contains $\beta_{g}$ with multiplicity $m+n$. Thus, fixing $\mathbf{y}_{0}, \Theta_{\delta \beta}$, and $\mathbf{w}_{0}$ in $\mathbb{T}_{\alpha} \cap \mathbb{T}_{\delta}, \mathbb{T}_{\delta} \cap \mathbb{T}_{\beta}$ and $\mathbb{T}_{\alpha} \cap \mathbb{T}_{\beta}$ respectively, the function

$$
m_{p}: \pi_{2}\left(\mathbf{y}_{0}, \Theta_{\delta \beta}, \mathbf{w}_{0}\right) \longrightarrow \mathbb{Z} / m \mathbb{Z}
$$

descends to a function

$$
\mathfrak{m}: \operatorname{Spin}^{c}\left(W_{n+m}^{\prime}(K)\right) \longrightarrow \mathbb{Z}
$$

with the property that

$$
\mathfrak{m}(\mathfrak{s}-\operatorname{PD}[\widehat{F}])=\mathfrak{m}(\mathfrak{s})+m+n .
$$

Note that $\mathfrak{m}$ depends on the choice of $p$ and the initial fixed intersection points up to an overall additive constant; there is also an orientation issue here which is a matter of convention. Composing with the isomorphism from (7), it follows at once that

$$
\theta \circ f_{2}^{+}=\sum_{\mathfrak{s} \in \operatorname{Spin}^{c}\left(W_{n+m}^{\prime}(K)\right)} T^{\mathfrak{m}(\mathfrak{s})} \cdot f_{W_{n+m}^{\prime}}^{+}(K), \mathfrak{s} .
$$

We must check that $f_{2}^{+} \circ f_{1}^{+} \simeq 0$. This is proved as usual using associativity for holomorphic triangles. The verification involves a calculation in the Heegaard triple $(\Sigma, \boldsymbol{\gamma}, \boldsymbol{\delta}, \boldsymbol{\beta}, z)$, showing that the pairing of $\Theta_{\gamma \delta}$ and $\Theta_{\delta \beta}$ in $H F^{\leq 0}\left(Y_{\gamma, \beta} ; \mathbb{Z}[\mathbb{Z} / m \mathbb{Z}]\right)$ vanishes; ie

$$
\sum_{\mathbf{x} \in \mathbb{T}_{\gamma} \cap \mathbb{T}_{\beta}} \sum_{\left\{\psi \in \pi_{2}\left(\Theta_{\gamma \delta}, \Theta_{\delta \beta}, \mathbf{x}\right) \mid \mu(\psi)=0\right\}} \# \mathcal{M}(\psi) \cdot\left[\mathbf{x},-n_{z}(\psi)\right] \cdot T^{m_{p}(\psi)}
$$

is null-homologous.

The claim amounts to showing that triangles come in cancelling pairs with the same value of $n_{z}(\psi)$ and $T$ power. The fact that any two triangles with the same value of $n_{z}(\psi)$ connecting $\Theta_{\gamma \delta}, \Theta_{\delta \beta}$, and $\Theta_{\gamma \beta}$ have the same power of $T$ follows from the fact that a generator for the space of the triply-periodic domains for the Heegaard triple $(\Sigma, \boldsymbol{\gamma}, \boldsymbol{\delta}, \boldsymbol{\beta}, z)$ has $m_{p}(\psi)=m$, and of course any two triangles with the same value of $n_{z}(\psi)$ differ by a triply-periodic domain. Next, we claim that triangles with a fixed value of $n_{z}(\psi)$ come in pairs. This is straightforward to verify - in fact, a linear map transforms the problem into the same lattice point count considered in the integer surgeries long exact sequence, [15, Theorem 10.19]. The usual proof of the associativity law for maps induced by triangles now gives a null-homotopy $H_{1}^{+}: C F^{+}\left(Y_{n}(K)\right) \longrightarrow C F^{+}(Y ; \mathbb{Z}[\mathbb{Z} / m \mathbb{Z}])$ of the composite $f_{2}^{+} \circ f_{1}^{+}$, defined by counting pseudo-holomorphic quadrilaterals. 
One could now establish existence of a long exact sequence in homology following the outline of Ozsváth-Szabó ([15] or [19]). Following the approach of [15], observe that there are curves in the isotopy class of $\delta_{g}$ which approximate arbitrarily well the juxtaposition of curves $m \cdot \beta_{g}+\gamma_{g}$. The map from $C F^{+}\left(Y_{n}(K)\right)$ into $C F^{+}\left(Y_{m+n}(K)\right)$ is injective, since the counts of small triangles (with vertex at the canonical $\operatorname{Spin}^{c}$ structure) induce the nearest point map from $C F^{+}\left(Y_{n}(K)\right)$ into $C F^{+}\left(Y_{m+n}(K)\right)$, while the counts of small triangles with vertex at $\beta_{g} \cap \gamma_{g}$ and reference point $p \in \beta_{g}$ induces the nearest point map tensored with a surjection onto $\mathbb{Z} / m \mathbb{Z}$.

However, for the stronger statement about the quasi-isomorphisms, we need to use the argument from [19, Section 4], and in particular, we need to place the third map on equal footing with the first two.

To this end, let $c \in \mathbb{Z} / m \mathbb{Z}$ be the element gotten as follows. Fix any $\psi \in \pi_{2}\left(\Theta_{\gamma \beta}, \Theta_{\beta \delta}, \Theta_{\gamma \delta}\right)$, where the $\Theta_{i, j}$ have been chosen before (recall that $\Theta_{\gamma \delta}$ represents the generator of $H F^{\leq 0}\left(L(m, 1) \#\left(\#^{g-1}\left(S^{2} \times S^{1}\right)\right), \ell\right)$ in the canonical $\operatorname{Spin}^{c}$ structure). Observe that the congruence class of $m_{p}(\psi)$ modulo $m$ is independent of the choice of $\psi$ (as any two such $\psi$ differ by a triply-periodic domain, whose multiplicity at $p$ is some multiple of $m$ ). Denote this quantity by $c \in \mathbb{Z} / m \mathbb{Z}$. (It, of course, depends on the particular Heegaard triple.)

Define

$$
f_{3}^{+}: C F^{+}(Y ; \mathbb{Z}[\mathbb{Z} / m \mathbb{Z}]) \longrightarrow C F^{+}\left(Y_{n}(K)\right)
$$

by the formula

$$
f_{3}^{+}\left(T^{s} \cdot[\mathbf{x}, i]\right)=\sum_{\mathbf{y} \in \mathbb{T}_{\alpha} \cap \mathbb{T}_{\gamma}} \sum_{\substack{\mu(\psi)=0,\left\{\psi \in \pi_{2}\left(\mathbf{x}, \Theta_{\beta \gamma}, \mathbf{y}\right)\right.}} \quad \# \mathcal{M}(\psi) \cdot\left[\mathbf{y}, i-n_{z}(\psi)\right] .
$$

More invariantly, we can think of $f_{3}^{+}$as a sum of maps induced by the two-handle cobordism $W_{n}(K): Y \longrightarrow Y_{n}(K)$. Specifically, multiplicity at $p$ induces a map

$$
\mathfrak{m}^{\prime}: \operatorname{Spin}^{c}\left(W_{n}(K)\right) \longrightarrow \mathbb{Z}
$$

with the property that $\mathfrak{m}^{\prime}(\mathfrak{s}-\operatorname{PD}[\widehat{F}])=\mathfrak{m}^{\prime}(\mathfrak{s})+n$. Now, there is some $c^{\prime} \in \mathbb{Z} / m \mathbb{Z}$ with the property that

$$
f_{3}^{+} \circ \theta\left(T^{s} \cdot \xi\right)=\sum_{\left\{\mathfrak{s} \in \operatorname{Spin}^{c}\left(W_{n}(K)\right) \mid \mathfrak{m}^{\prime}(\mathfrak{s})+c^{\prime} \equiv s\right.} f_{W_{n}(K), \mathfrak{s}}^{+} .
$$

The verifications that $f_{3}^{+} \circ f_{2}^{+} \simeq 0$ and $f_{1}^{+} \circ f_{3}^{+} \simeq 0$ proceed as before. In fact, counts of quadrilaterals define null-homotopies $\mathrm{H}_{i}^{+}$of $f_{i+1}^{+} \circ f_{i}^{+}$.

Algebraic $8 \mathcal{G}$ Geometric Topology, Volume 8 (2008) 
For the quasi-isomorphism statement, we count pseudo-holomorphic quadrilaterals for Heegaard quadruples. Let $\boldsymbol{\beta}^{\prime}, \boldsymbol{\gamma}^{\prime}$, and $\boldsymbol{\delta}^{\prime}$ denote suitable exact Hamiltonian translates of the corresponding curves $\boldsymbol{\beta}, \boldsymbol{\gamma}$, and $\delta$ respectively. It is easy to see that for the resulting Heegaard tuples $\left(\Sigma, \boldsymbol{\gamma}, \boldsymbol{\delta}, \boldsymbol{\beta}, \boldsymbol{\gamma}^{\prime}, z\right)$, and $\left(\Sigma, \boldsymbol{\delta}, \boldsymbol{\beta}, \boldsymbol{\gamma}, \boldsymbol{\delta}^{\prime}, z\right)$, there is exactly one Whitney quadrilateral $\varphi$ connecting the $\Theta_{i, j}$ with $\mathcal{D}(\varphi) \geq 0$, $n_{z}(\varphi)=0$, and $\mu(\phi)=-1$. (Here, $\mathcal{D}(\varphi)$ denotes the set of local multiplicities of $\varphi$ on $\Sigma$, cf [16]; these local multiplicities must be non-negative for the homotopy class to admit pseudo-holomorphic representatives). Moreover, this homotopy class admits a unique pseudo-holomorphic representative, compare [19, Section 4]. This is a routine adaptation of the arguments given in that section. For the Heegaard tuple $\left(\Sigma, \boldsymbol{\gamma}, \boldsymbol{\delta}, \boldsymbol{\beta}, \boldsymbol{\gamma}^{\prime}, z\right)$, it follows that under the map induced by counting holomorphic quadrilaterals, the map

$$
\widehat{H F}\left(\mathbb{T}_{\gamma}, \mathbb{T}_{\delta}\right) \otimes \widehat{H F}\left(\mathbb{T}_{\delta}, \mathbb{T}_{\beta}\right) \otimes \widehat{H F}\left(\mathbb{T}_{\beta}, \mathbb{T}_{\gamma^{\prime}}\right) \longrightarrow \widehat{H F}\left(\mathbb{T}_{\gamma}, \mathbb{T}_{\gamma^{\prime}}\right)
$$

carries the top-dimensional generator of the left-hand-side to the top-dimensional generator of the right-hand-side cf [19, (11)]. A corresponding statement for $H F=0$ (taken with coefficients in the ring of formal power series in $U, \mathbb{Z}[[U]]$ ) is now a formal consequence, showing that the top-dimensional generator of the tensor product of $H F^{\leq 0}$ is mapped to a multiple of the generator of $H F^{\leq 0}\left(\mathbb{T}_{\gamma}, \mathbb{T}_{\gamma^{\prime}}\right)$ by a unit in $\mathbb{Z}[[U]]$. Corresponding remarks apply to the quadruple $\left(\Sigma, \boldsymbol{\delta}, \boldsymbol{\beta}, \boldsymbol{\gamma}, \boldsymbol{\delta}^{\prime}, z\right)$.

The Heegaard tuple $\left(\Sigma, \boldsymbol{\beta}, \boldsymbol{\gamma}, \boldsymbol{\delta}, \boldsymbol{\beta}^{\prime}\right)$ works slightly differently, as now we are required to take twisted coefficients when using the pair $\boldsymbol{\beta}$ and $\boldsymbol{\beta}^{\prime}, \widehat{H F}\left(\mathbb{T}_{\beta}, \mathbb{T}_{\beta^{\prime}}, \mathbb{Z}[\mathbb{Z} / m \mathbb{Z}]\right)$. In this case, the generator of the top-dimensional homology group is not represented by the intersection point $\Theta_{\beta, \beta^{\prime}}$, but rather by the element $\left(\sum_{i=0}^{m-1} T^{i}\right) \cdot \Theta_{\beta, \beta^{\prime}}$. Correspondingly, there are now $m$ different homotopy quadrilaterals $\varphi_{i}$ with $i=0, \ldots, m-1$ which have $\mathcal{D}\left(\varphi_{i}\right) \geq 0, n_{z}(\varphi)=0$, and $\mu(\varphi)=-1$. We can order these so that $m_{p}\left(\varphi_{i}\right)=i$. Each of these has a unique holomorphic representative. Again, this shows that the map induced by counting holomorphic quadrilaterals (and recording their multiplicity at $p$ in the exponent of a formal variable $T$ )

$$
\widehat{H F}\left(\mathbb{T}_{\beta}, \mathbb{T}_{\gamma}\right) \otimes \widehat{H F}\left(\mathbb{T}_{\gamma}, \mathbb{T}_{\delta}\right) \otimes \widehat{H F}\left(\mathbb{T}_{\delta}, \mathbb{T}_{\beta}\right) \longrightarrow \widehat{H F}\left(\mathbb{T}_{\beta}, \mathbb{T}_{\beta^{\prime}} ; \mathbb{Z}[\mathbb{Z} / m \mathbb{Z}]\right)
$$

carries the top-dimensional generator of the left-hand-side to $\left(\sum_{i=0}^{m-1} T^{i}\right) \cdot \Theta_{\beta, \beta^{\prime}}$, the top-dimensional generator of the right-hand-side. Corresponding remarks for the maps induced on $H F^{\leq 0}$ follow at once.

With this said, the argument from [19] prove that the chain maps

$$
\phi^{+}: C F^{+}\left(Y_{n}(K)\right) \longrightarrow M\left(f_{2}^{+}\right) \text {and } \psi^{+}: M\left(f_{2}^{+}\right) \longrightarrow C F^{+}\left(Y_{n}(K)\right)
$$


defined by

$$
\phi^{+}(\xi)=\left(f_{1}^{+}(\xi), H_{1}^{+}(\xi)\right)
$$

and

$$
\psi^{+}(x, y)=H_{2}^{+}(x)+f_{3}^{+}(y)
$$

respectively are quasi-isomorphisms. (Observe, incidentally, that in [19], we gave a proof using $\widehat{C F}$. To pass from there to $C F^{+}$, observe that the chain maps and chain homotopies used are all $\mathbb{Z}[U]$-equivariant maps; and hence the chain maps above are in fact $\mathbb{Z}[U]$-equivariant quasi-isomorphisms.)

\section{Proof of Theorem 1.1}

Before proving Theorem 1.1, we give a more precise statement.

Let $Y$ be an integral homology three-sphere, equipped with a knot $K \subset Y$. Fix also an integer $n \neq 0$.

In the introduction, we considered chain complexes $\mathbb{A}^{+}=\bigoplus_{s \in \mathbb{Z}} A_{s}^{+}$and $\mathbb{B}^{+}=$ $\bigoplus_{s \in \mathbb{Z}} B_{s}^{+}$and a chain map $D_{n}^{+}: \mathbb{A}^{+} \longrightarrow \mathbb{B}^{+}$defined by $D_{n}^{+}\left(\left\{a_{s}\right\}_{s \in \mathbb{Z}}\right)=\left\{b_{s}\right\}_{s \in \mathbb{Z}}$, where here $b_{s}=h_{s-n}^{+}\left(a_{s-n}\right)+v_{s}^{+}\left(a_{s}\right)$. We refine this further as follows.

For each $i \in \mathbb{Z} / n \mathbb{Z}$, let

$$
\mathbb{A}_{i}^{+}=\bigoplus_{\{s \in \mathbb{Z} \mid s \equiv i(\bmod n)\}} A_{s}^{+},
$$

and

$$
\mathbb{B}_{i}^{+}=\bigoplus_{\{s \in \mathbb{Z} \mid s \equiv i(\bmod n)\}} B_{s}^{+} .
$$

There is a splitting

$$
\mathbb{A}^{+}=\bigoplus_{i \in \mathbb{Z} / n \mathbb{Z}} \mathbb{A}_{i}^{+} \text {and } \mathbb{B}^{+}=\bigoplus_{i \in \mathbb{Z} / n \mathbb{Z}} \mathbb{B}_{i}^{+}
$$

and the restriction of $D_{n}^{+}$to $\mathbb{A}_{i}^{+}$can be thought of as a map

$$
D_{n, i}^{+}: \mathbb{A}_{i}^{+} \longrightarrow \mathbb{B}_{i}^{+}
$$

Moreover, the mapping cone $\mathbb{X}^{+}(n)$ of $D_{n}^{+}$splits into a direct sum of mapping cones $\mathbb{X}^{+}(n)=\bigoplus_{i \in \mathbb{Z} / n \mathbb{Z}} \mathbb{X}_{i}^{+}(n)$, where here $\mathbb{X}_{i}^{+}(n)$ is the mapping cone of $D_{n, i}^{+}$. 
Clearly, $\mathbb{X}^{+}(n)$ can be given a relative $\mathbb{Z}$-grading, which is compatible with the relative $\mathbb{Z}$ grading on $A_{s}^{+}$and $B_{s}^{+}$, and with the property that $v^{+}$and $h^{+}$(thought of as endomorphisms of $\left.\mathbb{X}^{+}(n)\right)$ are homogeneous maps of degree -1 .

More explicitly, suppose that $n>0$, and consider the absolute grading on $C F^{+}(Y)$. Although each $B_{s}^{+} \cong C F^{+}(Y)$, we shift the grading; ie writing $s=\sigma+\ell \cdot n$ where $\sigma$ is the representative for $i(\bmod n)$ with $0 \leq \sigma<n$. We lift the natural relative $\mathbb{Z}$-grading on $B_{s}^{+}$so that the homogeneous elements of $C F^{+}(Y)$ of degree $d$ correspond to the homogeneous elements of $B_{\sigma+\ell \cdot n}^{+}$of degree $d+2 \ell \sigma+n \ell(\ell-1)-1$. We claim we can then consistently shift the gradings on $A_{s}^{+}$so that both maps $v_{s}^{+}: A_{s}^{+} \longrightarrow B_{s}^{+}$ and $h_{s}^{+}: A_{s}^{+} \longrightarrow B_{s+n}^{+}$are homogeneous maps of degree -1 . This gives a grading on $\mathbb{X}_{i}^{+}(n)$.

When considering $\mathbb{X}^{+}(-n)$ (where here $n>0$ ), we still have that $B_{s}^{+} \cong C F^{+}(Y)$. Writing $s=-(\sigma+\ell \cdot n)$, for $0 \leq \sigma \leq n$, we identify homogeneous elements of degree $d$ in $C F^{+}(Y)$ with the homogeneous elements of $B_{-\sigma-\ell \cdot n}^{+}$of degree $d-2 \ell \sigma-n \ell(\ell-1)$. We can then consistently shift the gradings on $A_{s}^{+}$so that both maps $v_{s}^{+}: A_{s}^{+} \longrightarrow B_{s}^{+}$ and $h_{s}^{+}: A_{s}^{+} \longrightarrow B_{s-n}^{+}$are homogeneous maps of degree -1 . This gives an integral grading on $\mathbb{X}_{i}^{+}(-n)$.

Theorem 4.1 Let $Y$ be an integral homology three-sphere. The homology of the mapping cone $\mathbb{X}^{+}(n)$ of

$$
D_{n}^{+}: \mathbb{A}^{+} \longrightarrow \mathbb{B}^{+}
$$

is isomorphic to $\mathrm{HF}^{+}\left(Y_{n}(K)\right)$. Moreover, the summand $\mathrm{HF}^{+}\left(Y_{n}(K), i\right)$ (under the identification $\operatorname{Spin}^{c}\left(Y_{n}(K)\right) \cong \mathbb{Z} / n \mathbb{Z}$ given in Section 2.4) is identified with the homology of the summand $\mathbb{X}_{i}^{+}(n)$. Also, this map identifies the relative $\mathbb{Z}$-grading on $\mathrm{HF}^{+}\left(Y_{n}(K), i\right)$ with the one described above on $H_{*}\left(\mathbb{X}_{i}^{+}(n)\right)$. In fact, the isomorphism from $H_{*}\left(\mathbb{X}_{i}^{+}(n)\right)$ to $H F^{+}\left(Y_{n}(K), i\right)$ is a homogeneous map of degree $d(n, i)$, as defined in (2).

The following result is a quick consequence of the proof of Theorem 4.1. It shows that knot Floer homology also determines the maps induced by the natural cobordisms from $Y$ to $Y_{n}(K)$.

Theorem 4.2 The following square commutes:

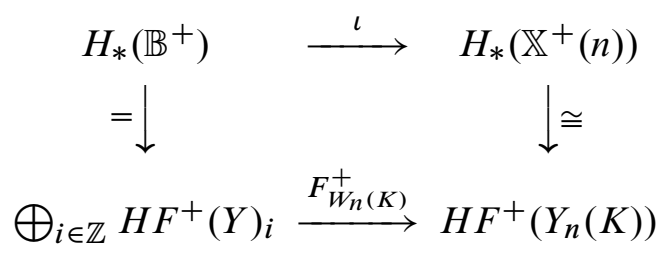

Algebraic 83 Geometric Topology, Volume 8 (2008) 
where the second vertical isomorphism is provided by Theorem 4.1, the top horizontal map is the natural map induced from the mapping cone construction, while the bottom one is the map induced by cobordisms; ie the $i$ th factor $\mathrm{HF}^{+}(Y)_{i}$ is mapped to $\mathrm{HF}^{+}\left(Y_{n}(K)\right)$ by the natural map induced by the two-handle cobordism $W_{n}(K)$ endowed with the $\operatorname{Spin}^{c}$ structure with

$$
\left\langle c_{1}(\mathfrak{s}),[\widehat{F}]\right\rangle+n=2 i .
$$

Note that Theorem 1.1 is a special case of Theorem 4.1 and Theorem 4.2.

Most of this section is devoted to a proof of Theorem 4.1. In Section 4.1, we set up some notation and algebraic preliminaries for the proof. The theorem follows by applying Theorem 3.1, choosing the surgery coefficient $m$ to be sufficiently large. Thus, in in Section 4.2, we study $\operatorname{Spin}^{c}$ structures over one of the cobordisms from Theorem 3.1 , when the surgery coefficient is large. This provides enough information to prove the special case of Theorem 1.1 for the case of $\widehat{H F}$, cf Section 4.3. We assume for simplicity that $n>0$ throughout most of the exposition, returning to the case where $n<0$ in a later subsection. The case for $H F^{\delta}$ (arbitrary $\delta \geq 0$ ) follows as well, with minor notational modifications, cf Section 4.4. In Section 4.5, we return to the question of $\operatorname{Spin}^{c}$ structures over cobordisms, when the surgery coefficient is sufficiently large. With this done, we can complete the the proof of Theorem 4.1, in the case where $n>0$. In Section 4.7, we describe the modifications needed to make the proof go over to the case where $n<0$. In Section 4.8, we make a few comments regarding the case where $n=0$. In Section 4.9, we give the proof of Theorem 4.2. In Section 4.10, we discuss some generalizations of Theorem 4.1.

\subsection{Truncation}

Although we are primarily interested in $H F^{+}$, we will find it convenient to use $H F^{\delta}$ at various times, and also easier to explain things in terms of $\widehat{H F}$. To this end, we will use some straightforward notational changes. Specifically, for any integer $\delta \geq 0$, let $A_{i}^{\delta}, B_{i}^{\delta}, \mathbb{A}_{i}^{\delta}$, and $\mathbb{B}_{i}^{\delta}$ denote the subsets of $A_{i}^{+}, B_{i}^{+}, \mathbb{A}_{i}^{+}$, and $\mathbb{B}_{i}^{+}$respectively which lie in the kernel of multiplication by $U^{\delta+1}$. Thus, the mapping cone $\mathbb{X}_{i}^{\delta}(n)$ of the restriction $D_{n, i}^{\delta}$ of $D_{n, i}^{+}$, viewed as a map

$$
D_{n, i}^{\delta}: \mathbb{A}_{i}^{\delta} \longrightarrow \mathbb{B}_{i}^{\delta},
$$

is the kernel of the action by $U^{\delta+1}$ on $\mathbb{X}_{i}^{+}(n)$. Similarly, we let $\widehat{A}_{i}, \widehat{B}_{i}, \widehat{\mathbb{A}}_{i}, \widehat{\mathbb{B}}_{i}$ denote the corresponding groups $A_{i}^{\delta}, B_{i}^{\delta}, \mathbb{A}_{i}^{\delta}$, and $\mathbb{B}_{i}^{\delta}$ with $\delta=0$. 
It will be convenient for us also to truncate the infinite constructions from the introduction. Specifically, for fixed integers $b$, let

$$
\begin{aligned}
& \mathbb{A}_{i}^{+}(b)=\underset{\left\{s \in \mathbb{Z} \mid s \equiv i \quad{ }_{(\bmod n),-b \leq s \leq b\}}\right.}{A_{s}^{+} \subset \mathbb{A}_{i}^{+}} \\
& \mathbb{B}_{i}^{+}(b)=\bigoplus_{\{s \in \mathbb{Z} \mid s \equiv i} \bigoplus_{(\bmod n),-b+n \leq s \leq b\}}^{+} \subset \mathbb{B}_{i}^{+} .
\end{aligned}
$$

Similarly, we let $D_{n, i ; b}^{+}: \mathbb{A}^{+}(b) \longrightarrow \mathbb{B}^{+}(b)$ denote the restriction of $D_{n, i}^{+}$, and let and $\mathbb{X}_{i}^{+}(n ; b) \subset \mathbb{X}_{i}^{+}(n)$ denote the corresponding subset of the mapping cone.

Note that when $n>0$, there is a quotient map $Q_{b}^{+}: \mathbb{X}_{i}^{+}(n) \longrightarrow \mathbb{X}_{i}^{+}(n ; b)$; and also $\widehat{Q}_{b}$ and $Q_{b}^{\delta}$ (for any $\delta \geq 0$ ) using corresponding constructions on $\widehat{\mathbb{X}}_{i}(n)$ and $\mathbb{X}_{i}^{\delta}(n)$. When $n<0$, there are inclusion maps $i_{b}^{+}: \mathbb{X}_{i}^{+}(n ; b) \longrightarrow \mathbb{X}_{i}^{+}(n)$ and also $\widehat{i}_{b}$ and $i_{b}^{\delta}$ for the other variants.

Lemma 4.3 If $b$ is sufficiently large, then when $n>0$, the quotient maps $Q_{b}^{+}, \widehat{Q}_{b}$ and $Q_{b}^{\delta}$ are quasi-isomorphisms; when $n<0$, the inclusions $i_{b}^{+}, \widehat{i}_{b}$, and $i_{b}^{\delta}$ are quasi-isomorphisms.

Proof This follows quickly from the fact that for all $s$ sufficiently large, the maps $v_{s}^{+}$ (and $\widehat{v}_{s}, v_{s}^{\delta}$ ) and $h_{-s}^{+}$(and $\widehat{h}_{-s}, h_{-s}^{\delta}$ ) are isomorphisms.

It follows easily from the above that $\mathbb{X}_{i}^{+}(n)$ is of $C F^{+}$-type, in the sense of Definition 2.6.

\subsection{Gradings}

Let $Y$ be a three-manifold with a null-homologous $K \subset Y$. Continuing notation from Section 2.4, given an integer $N$, let $W_{N}^{\prime}(K): Y_{N}(K) \longrightarrow Y$ denote the induced two-handle cobordism. Fix also Seifert surface $F$ for $K$, and let $\widehat{F} \subset W_{N}^{\prime}(K)$ denote the surface obtained by capping off $F$ in $W_{N}^{\prime}(K)$.

In the following statement, note that we continue conventions from Section 2.3: $C F^{\delta}\left(Y_{N}(K),[i]\right)$ and $C F^{\delta}(Y)$ will denote complexes which are quasi-isomorphic to the Heegaard Floer homology complexes for some choice of Heegaard diagram and auxilliary data. 
Lemma 4.4 Suppose that $K$ is a knot in a three-manifold $Y$. Then, for any constant $\delta \geq 0$, there is another constant $b$ with the following property. For all sufficiently large $N$, for any fixed $[i] \in \mathbb{Z} / N \mathbb{Z}$, there are at most two $\operatorname{Spin}^{c}$ structures on $W_{N}^{\prime}(K)$ whose restriction to $Y_{N}(K)$ is $[i]$ with the property that

$$
\min \operatorname{gr} C F^{\delta}(Y)-\max \operatorname{gr} C F^{\delta}\left(Y_{N},[i]\right) \leq \frac{c_{1}(\mathfrak{s})^{2}+1}{4} ;
$$

these are the $\operatorname{Spin}^{c}$ structures with

$$
\left\langle c_{1}(\mathfrak{s}),[\widehat{F}]\right\rangle=2 s \pm N,
$$

where $s$ is an integer $\equiv i(\bmod N)$, and which satisfy $-N / 2 \leq s<N / 2$. All other $\operatorname{Spin}^{c}$ structures satisfy the inequality

$$
c_{1}(\mathfrak{s})^{2} \leq-4 N .
$$

Moreover, if any integral representative $s$ for $i$ satisfies $|s|>b$, then there is a unique Spin $^{c}$ structure satisfying (16), and it is the one for which $\left|\left\langle c_{1}(\mathfrak{s}),[\widehat{F}]\right\rangle\right|$ is minimal.

Proof The set of $\operatorname{Spin}^{c}$ structures over $W_{N}^{\prime}(K)$ can be identified with the set of integers via the correspondence which sends $\mathfrak{s}$ to the integer $s$ determined by the formula

$$
\left\langle c_{1}(\mathfrak{s}),[\widehat{F}]\right\rangle-N=2 s .
$$

Fixing the restriction of $\mathfrak{s}$ to $Y_{N}(K)$ corresponds to fixing the equivalence class of $s$ modulo $n$. Thus, maximizing the function $\left(c_{1}(\mathfrak{s})^{2}+1\right) / 4$ over the set of $\operatorname{Spin}^{c}$ structures whose restriction to $Y_{N}(K)$ corresponds to $i \in \mathbb{Z} / N \mathbb{Z}$ amounts to maximizing the quadratic function

$$
q(s)=\frac{1}{4}\left(1-\frac{(N+2 s)^{2}}{N}\right)
$$

over all integers $s$ with $s \equiv i(\bmod N)$.

This quadratic form is maximized by the representative $i$ for $[i]$ with $-N<i \leq 0$. The second largest value is smaller by $\min (2|i|, 2 N+2 i)$, and all other values are smaller than $q(i)$ by at least $\max (2|i|, 2 N+2 i)$. Thus, if $\mathfrak{s}$ is any $\operatorname{Spin}^{c}$ structure for which the quadratic function takes on neither its maximal or next-to-maximal value, we have that

$$
\frac{c_{1}(\mathfrak{s})^{2}+1}{4} \leq q(i)-\max (2|i|, 2 N+2 i) \leq q(i)-N \leq \frac{1}{4}-N .
$$

Algebraic 83 Geometric Topology, Volume 8 (2008) 
On the other hand, according to Corollary 2.4, there is a constant $C_{2}$ with the property that if we let $\Delta=\min \operatorname{gr} C F^{\delta}(Y)-\max \operatorname{gr} C F^{\delta}\left(Y_{N},[i]\right)$, then

$$
q(i) \leq \Delta+C_{2} .
$$

Thus, if $N$ is sufficiently large, inequality (16) is violated.

Moreover, for the final statement, observe that the hypothesis that any representative $s$ for $i$ satisfies $|s|>b$ is equivalent to the assertion that $\min (2|i|, 2(N+i))>2 b$. Moreover, if $\mathfrak{s}$ is any $\operatorname{Spin}^{c}$ structure for which the quadratic form is not minimized,

$\frac{c_{1}(\mathfrak{s})^{2}+1}{4} \leq q(i)-\min (2|i|, 2 N+2 i) \leq \Delta+C_{2}-\min (2|i|, 2 N+2 i)<\Delta+C_{2}-2 b ;$

thus the choice of $b=C_{2} / 2$ satisfies the final assertion.

\subsection{The case of $\widehat{H F}$}

Theorem 1.1 follows from an analysis of Theorem 3.1 for a suitable choice of $m-$ specifically, we choose $m=n k$ where $k$ is a sufficiently large positive integer. We shall assume for the time being that $n>0$, returning to the case of negative surgery coefficients in Section 4.7. Our aim in the present subsection is to prove an analogue of Theorem 1.1 for $\widehat{C F}$, showing that $\widehat{\mathbb{X}}_{i}(n)$ is quasi-isomorphic to $\widehat{C F}\left(Y_{n}(K), i\right)$. (However, the statement about maps induced by cobordisms will be relegated to Section 4.9 below, and the case where $n<0$ is handled in Section 4.7.)

Let

$$
\widehat{f}_{1}: \widehat{C F}\left(Y_{n}(K)\right) \longrightarrow \widehat{C F}\left(Y_{n(k+1)}(K)\right)
$$

and

$$
\widehat{f}_{2}: \widehat{C F}\left(Y_{n(k+1)}(K)\right) \longrightarrow \widehat{C F}(Y ; \mathbb{Z}[\mathbb{Z} / n k \mathbb{Z}])
$$

denote the maps induced by $f_{1}^{+}$and $f_{2}^{+}$from (8) and (9) on $\widehat{C F} \subset C F^{+}$, thought of as the kernel of multiplication by $U$. We will also fix an identification of chain complexes

$$
\widehat{C F}(Y ; \mathbb{Z}[\mathbb{Z} / n k \mathbb{Z}]) \cong \widehat{C F}(Y) \otimes_{\mathbb{Z}} \mathbb{Z}[\mathbb{Z} / n k \mathbb{Z}],
$$

and so as to think of $\widehat{C F}(Y ; \mathbb{Z}[\mathbb{Z} / n k \mathbb{Z}])$ as a direct sum of complexes

$$
\widehat{C F}(Y ; \mathbb{Z}[\mathbb{Z} / n k \mathbb{Z}]) \cong \bigoplus_{s \in \mathbb{Z} / n k \mathbb{Z}} T^{s} \otimes \widehat{C F}(Y)
$$

(We are using here an identification $\theta$ as in (7), which is well-defined up to an overall shift by $T^{c}$ for some constant $c$ which we will fix later. We suppress the identification $\theta$ to simplify notation.) 
Let $W_{n(k+1)}^{\prime}(K)$ be the natural two-handle cobordism from $Y_{n(k+1)}(K)$ to $Y$. We abbreviate this cobordism by $W^{\prime}(k)$. Recall that a choice of Seifert surface $F$ for $K$ gives rise to a closed surface $\widehat{F} \subset W^{\prime}(k)$ with

$$
[\widehat{F}] \cdot[\widehat{F}]=-n(k+1) .
$$

Given $s \in \mathbb{Z}$, let $\widehat{\mathfrak{A}}_{s}=\widehat{C F}\left(Y_{n(k+1)}(K), s\right)$; ie it is the summand of $\widehat{C F}\left(Y_{n(k+1)}(K)\right)$ in a $\operatorname{Spin}^{c}$ structure gotten by restricting a $\operatorname{Spin}^{c}$ structure $\mathfrak{s}$ over $W^{\prime}(k)$ which satisfies

$$
\left\langle c_{1}(\mathfrak{s}) \cdot[\widehat{F}]\right\rangle-n(k+1) \equiv 2 s \quad(\bmod 2 n(k+1)) .
$$

Moreover, let $\mathfrak{x}_{s}$ resp. $\mathfrak{y}_{s}$ denote the $\operatorname{Spin}^{c}$ structures over the cobordism

$$
W^{\prime}(k): Y_{n(k+1)}(K) \longrightarrow Y
$$

which satisfy

$$
\left\langle c_{1}\left(\mathfrak{x}_{s}\right),[\widehat{F}]\right\rangle+n(k+1)=2 s \text { and }\left\langle c_{1}\left(\mathfrak{y}_{s}\right),[\widehat{F}]\right\rangle-n(k+1)=2 s
$$

respectively.

For any $s$ in

$$
-\frac{n(k+1)}{2} \leq s<\frac{n(k+1)}{2},
$$

then $\mathfrak{x}_{s}$ and $\mathfrak{y}_{s}$ are the two $\operatorname{Spin}^{c}$ structures over $W^{\prime}(k)$ with fixed restriction to $Y_{n(k+1)}(K)$ for which the function $\frac{c_{1}(\mathfrak{s})^{2}+1}{4}$ takes on its two largest values (cf Lemma 4.4).

Observe that $\mathfrak{x}_{s}+\operatorname{PD}[\widehat{F}]=\mathfrak{y}_{s}$, and hence

$$
\mathfrak{m}\left(\mathfrak{x}_{s}\right)-\mathfrak{m}\left(\mathfrak{y}_{s}\right)=-n(k+1) \equiv-n \quad(\bmod n k),
$$

and also

$$
\mathfrak{m}\left(\mathfrak{x}_{s+n}\right)-\mathfrak{m}\left(\mathfrak{x}_{s}\right) \equiv n \quad(\bmod n k) .
$$

Thus, if we write

$$
\widehat{\mathfrak{B}}_{s}=T^{s} \otimes \widehat{C F}(Y) \subset \widehat{C F}(Y ; \mathbb{Z} / m \mathbb{Z})
$$

(with respect to the direct sum decomposition of (18)), then, after multiplying $f_{2}^{+}$with an overall factor of $T^{c}$ for some constant $c$, the components $\widehat{f}_{2}$ corresponding to the $\operatorname{Spin}^{c}$ structures $\mathfrak{x}_{s}$ and $\mathfrak{y}_{s}$ for integers $s$ in the range of inequality (19) give maps

$$
\widehat{\mathfrak{v}}_{s}: \widehat{\mathfrak{A}}_{s} \longrightarrow \widehat{\mathfrak{B}}_{s} \text { and } \quad \widehat{\mathfrak{h}}_{s}: \widehat{\mathfrak{A}}_{s} \longrightarrow \widehat{\mathfrak{B}}_{s+n}
$$

respectively. 
Now, apply Lemma 4.4 in the case where $\delta=0$, so that $C F^{\delta}=\widehat{C F}$, and choose $s$ to satisfy inequality (19). In this case, for all sufficiently large $k$, the lemma combined with the dimension shift formula (1) proves that $\mathfrak{x}_{s}$ and $\mathfrak{y}_{s}$ are the only two $\operatorname{Spin}^{c}$ structures which may induce non-trivial maps from $\widehat{C F}\left(Y_{n(k+1)}(K), s\right)$ into $\widehat{C F}(Y)$.

In sum, we have identified $\widehat{f}_{2}$, the map on $\widehat{C F}$ induced from the map $f_{2}^{+}$from (9), with the map

$$
\widehat{f}_{2} \bigoplus_{s \in \mathbb{Z} / n(k+1) \mathbb{Z}} \widehat{\mathfrak{A}}_{s} \longrightarrow \bigoplus_{s \in \mathbb{Z} / n k \mathbb{Z}} \widehat{\mathfrak{B}}_{s}
$$

induced by adding all the $\widehat{\mathfrak{v}}_{s}$ and $\widehat{\mathfrak{h}}_{s}$ in the range specified (19) (compare (10)).

Moreover, according to the second statement in Lemma 4.4, there is an integer $b$ (independent of $k$ ) with the property that for all $s \geq b$, we have that

$$
\widehat{\mathfrak{v}}_{-s}: \widehat{\mathfrak{A}}_{-s} \longrightarrow \widehat{\mathfrak{B}}_{-s} \text { and } \widehat{\mathfrak{h}}_{s}: \widehat{\mathfrak{A}}_{s} \longrightarrow \widehat{\mathfrak{B}}_{s+n}
$$

are null-homotopic. This, together with the integer surgeries long exact sequence (and the fact that $H F^{+}\left(S_{0}^{3}(K), s\right)=0$ for $s \geq b$ for some $\left.b\right)$, also shows that

$$
\widehat{\mathfrak{v}}_{s}: \widehat{\mathfrak{A}}_{s} \longrightarrow \widehat{\mathfrak{B}}_{s} \text { and } \widehat{\mathfrak{h}}_{-s}: \widehat{\mathfrak{A}}_{-s} \longrightarrow \widehat{\mathfrak{B}}_{-s+n}
$$

are quasi-isomorphisms. It follows that the mapping cone of $f_{2}^{\prime}$ is quasi-isomorphic to the mapping cone of

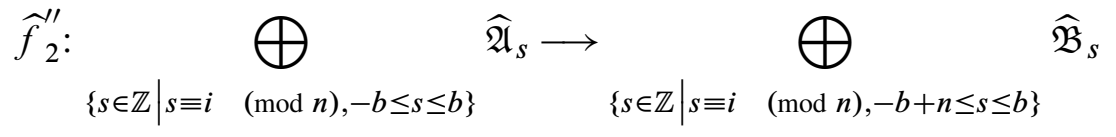

obtained by adding all the $\widehat{\mathfrak{v}}_{S}$ and $\widehat{\mathfrak{h}}_{S}$ in the given range.

Theorem 2.3 gives interpretations of these objects in terms of the knot Floer homology. Indeed, we have identifications (provided that $k$ is sufficiently large)

$$
\begin{aligned}
& \widehat{\mathfrak{A}}_{s} \stackrel{\widehat{\mathfrak{v}}_{s}}{\longrightarrow} \widehat{\mathfrak{B}}_{s} \quad \widehat{\mathfrak{A}}_{s} \stackrel{\widehat{\mathfrak{h}}_{s}}{\longrightarrow} \widehat{\mathfrak{B}}_{s+n} \\
& \Psi_{n(k+1), s}^{+} \downarrow \quad \downarrow \text { and } \downarrow \Psi_{n(k+1), s}^{+} \downarrow \\
& \widehat{A}_{s} \stackrel{\widehat{v}_{s}}{\longrightarrow} B_{s} \quad \widehat{A}_{s} \stackrel{\widehat{h}_{s}}{\longrightarrow} B_{s+n} .
\end{aligned}
$$

This in turn shows that the mapping cone of $\widehat{f}_{2}^{\prime \prime}$ is quasi-isomorphic to the mapping cone of

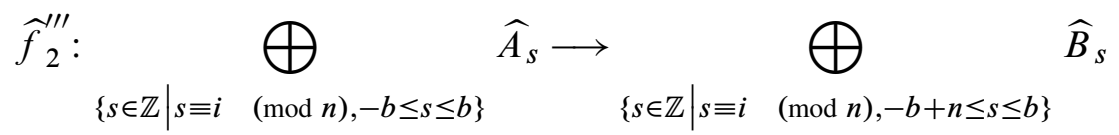


obtained by adding $\widehat{v}_{s}$ and $\widehat{h}_{S}$ in the given range (cf Lemma 2.1). But this mapping cone is identified the truncation $\widehat{\mathbb{X}}_{i}(n, b) \subset \mathbb{X}_{i}^{+}(n, b)$ from Section 4.1. Finally, applying Lemma 4.3 , we see that $\widehat{C F}\left(Y_{n}, i\right)$ is quasi-isomorphic to the mapping cone $\widehat{\mathbb{X}}_{i}(n)$.

\subsection{The case of $C F^{\delta}$}

A direct application of the argument from Section 4.3 proves the following proposition.

Proposition 4.5 Fix integers $n>0$ and $\delta \geq 0$. Then, there is a constant $b$ with the property that $C F^{\delta}\left(Y_{n}(K), i\right)$ is quasi-isomorphic to $\mathbb{X}_{i}^{\delta}(n ; b)$ (in the notation of Section 4.1).

Proof Apply the proof of Section 4.3, only now apply Lemma 4.4 with $\delta \geq 0$ arbitrary (rather than $=0$ ).

Of course, according to Lemma 4.3, the truncation is unnecessary, and we see that $H F^{\delta}\left(Y_{n}(K), i\right) \cong H_{*}\left(\mathbb{X}_{i}^{\delta}(n)\right)$. Moreover, when working with Floer homology with coefficients in a field, this statement for all $\delta \geq 0$ suffices to prove a version of Theorem 1.1 in an ungraded sense. We do not pursue this direction, but instead turn to gradings to establish the stronger form of the result stated in Theorem 4.1.

\subsection{More gradings}

We turn our attention now to the map $f_{1}^{+}$from (8), gotten by counting holomorphic triangles in the four-manifold $X_{\alpha, \gamma, \delta}$ belonging to the Heegaard triple $(\Sigma, \boldsymbol{\alpha}, \boldsymbol{\delta}, \boldsymbol{\beta})$. This four-manifold has three boundary components,

$$
Y_{n}(K), \quad Y_{\gamma, \delta} \cong L(n k, 1) \#\left(\#^{g-1}\left(S^{2} \times S^{1}\right)\right), \text { and } \quad Y_{n(k+1)}(K) .
$$

We abbreviate this four-manifold by $X(k)$. We will always fix the canonical $\operatorname{Spin}^{c}$ structure over the boundary component $Y_{\gamma, \delta}$ (cf Definition 3.2).

For $s \in \mathbb{Z}$, let

$$
\Pi_{s}^{A}: C F^{+}\left(Y_{n(k+1)}(K)\right) \longrightarrow C F^{+}\left(Y_{n(k+1)}(K), s\right)
$$

denote the natural projection map. Consider the map

$$
f_{1}^{+}: C F^{+}\left(Y_{n}(K)\right) \longrightarrow C F^{+}\left(Y_{n(k+1)}(K)\right)=\bigoplus_{s \in \mathbb{Z} / n(k+1) \mathbb{Z}} C F^{+}\left(Y_{n(k+1)}(K), s\right),
$$

from (8) and let $f_{1}^{\delta}$ denote its restriction to $C F^{\delta}\left(Y_{n}(K)\right)$.

In this subsection, we prove the following result. 
Proposition 4.6 Fix an absolute lift of the relative $\mathbb{Z}$-grading on $C F^{+}\left(Y_{n}(K)\right)$, and an integer $\delta \geq 0$. There exists a constant $b$ so that, for all sufficiently large $k$, there are absolute lifts of the relative $\mathbb{Z}$-gradings on both $C F^{\delta}\left(Y_{n(k+1)}(K), s\right) \subset$ $C F^{\delta}\left(Y_{n(k+1)}(K)\right)$ and $T^{s} \otimes C F^{\delta}(Y) \subset C F^{\delta}(Y ; \mathbb{Z}[\mathbb{Z} / n k \mathbb{Z}])$ for all $|s| \leq b$, with the property that $\Pi_{s}^{A} \circ f_{1}^{\delta}$ and also the restriction of $f_{2}^{\delta}$ to $C F^{\delta}\left(Y_{n(k+1)}(K), s\right)$ have degree zero.

We give the proof after setting up some terminology and establishing a lemma.

Sometimes, we find it convenient to pass between the absolute $\mathbb{Q}$-gradings on $C F^{\delta}\left(Y_{n(k+1)}(K)\right), C F^{\delta}(Y ; \mathbb{Z}[\mathbb{Z} / n k \mathbb{Z}])$ and the absolute gradings induced from the above proposition (eg induced from the absolute $\mathbb{Q}$-grading on $C F^{\delta}\left(Y_{n}\right)$ ). We call the absolute $\mathbb{Q}$-grading from before the "old gradings", and we call the induced gradings the "new gradings".

Lemma 4.7 Fix a constant $C_{0}$. For all sufficiently large $k$, the following statement holds. Each $\operatorname{Spin}^{c}$ structure over $Y_{n(k+1)}(K)$, has at most one extension $\mathfrak{s}$ over $X(k)$ whose restriction to $Y_{\gamma, \delta}$ is the canonical $\operatorname{Spin}^{c}$ structure and for which

$$
C_{0} \leq c_{1}(\mathfrak{s})^{2}+n k
$$

Proof It is easy to see that a generator $\Sigma$ for $H_{2}(X(k) ; \mathbb{Z})$ has

$$
\Sigma^{2}=-n k(k+1) .
$$

Thus, if $\mathfrak{s}$ satisfies inequality (22), then $c_{1}(\mathfrak{s})=\alpha \cdot \operatorname{PD}[\Sigma]$, where $\alpha$ satisfies

$$
|\alpha| \leq \sqrt{\frac{n k-C_{0}}{n k(k+1)}} \leq \frac{1}{2}
$$

for all sufficiently large $k$.

Note that any other $\operatorname{Spin}^{c}$ structure which interpolates between the same two $\operatorname{Spin}^{c}$ structures on $Y_{n}$ and $Y_{n(k+1)}$ has the form $\mathfrak{s}+\ell \cdot \operatorname{PD}[\Sigma]$ for some integer $\ell \neq 0$; now,

$$
\begin{aligned}
\left(c_{1}(\mathfrak{s}+\ell \cdot \operatorname{PD}[\Sigma])\right)^{2}-c_{1}(\mathfrak{s})^{2} & =4\left(\ell^{2} \Sigma \cdot \Sigma+\ell \cdot\left\langle c_{1}(\mathfrak{s}),[\Sigma]\right\rangle\right) \\
& \leq-4 \ell^{2} n k(k+1)\left(1-\frac{1}{2 \ell}|\alpha|\right) \\
& \leq-2 n k(k+1)
\end{aligned}
$$

if $k$ is sufficiently large. In particular, for sufficiently large $k$, inequality (22) is violated. 
Proof of Proposition 4.6 Fix an absolute lift of the relative $\mathbb{Z}$-grading on $C F^{+}\left(Y_{n}(K)\right)$, and fix some integer $\delta \geq 0$. Observe that $f_{1}^{\delta}$ can be decomposed as a sum of homogeneous terms, indexed by $\operatorname{Spin}^{c}$ structures $\mathfrak{s}$ over the four-manifold $X(k)$ whose restriction to $Y_{\delta, \gamma}$ is the canonical $\mathrm{Spin}^{c}$ structure. Each term is homogeneous, and with respect to the natural $\mathbb{Q}$-gradings on $C F^{+}\left(Y_{n}(K)\right)$ and $C F^{+}\left(Y_{n(k+1)}(K)\right)$ (ie the "old gradings"), they are homogeneous of degree

$$
\frac{c_{1}(\mathfrak{s})^{2}+n k}{4}
$$

(compare (1); observe that we have a third boundary component in this four-manifold, where we use a fixed generator $\Theta_{\delta, \gamma}$ ). In view of Corollary 2.4 there are constants $C_{1}$ and $C_{2}$ with the property that for all sufficiently large $k$,

$$
\begin{aligned}
\min & \operatorname{gr} C F^{\delta}\left(Y_{n(k+1)}(K)\right)-\max \operatorname{gr} C F^{\delta}\left(Y_{n}(K)\right) \\
\geq & \min \operatorname{gr} C F^{\delta}\left(Y_{n(k+1)}(K)\right)-\max \operatorname{gr} C F^{\delta}\left(Y_{n(k+1)}(K)\right) \\
& +\max \operatorname{gr} C F^{\delta}\left(Y_{n(k+1)}(K)\right)-\min \operatorname{gr} C F^{\delta}(Y) \\
& +\min \operatorname{gr} C F^{\delta}(Y)-\max \operatorname{gr} C F^{\delta}\left(Y_{n}(K)\right) \\
\geq & -C_{1}-C_{2}+\min _{i \in \mathbb{Z} / n(k+1) \mathbb{Z}} d(n(k+1), i) \\
& +\min \operatorname{gr} C F^{\delta}(Y)-\max \operatorname{gr} C F^{\delta}\left(Y_{n}(K)\right) \\
\geq & -\frac{1}{4}-C_{1}-C_{2}+\min \operatorname{gr} C F^{\delta}(Y)-\max \operatorname{grC} F^{\delta}\left(Y_{n}(K)\right) .
\end{aligned}
$$

Applying Lemma 4.7 with

$$
C_{0}=-\left(1+4\left(C_{1}+C_{2}-\min \operatorname{gr} C F^{\delta}(Y)+\max \operatorname{gr} C F^{\delta}\left(Y_{n}\right)\right),\right.
$$

we see that for all sufficiently large $k$, there is at most one $\operatorname{Spin}^{c}$ structure which interpolates between given $\operatorname{Spin}^{c}$ structures on $C F^{\delta}\left(Y_{n}(K)\right)$ and $C F^{\delta}\left(Y_{n(k+1)}(K)\right)$ and which satisfies the inequality

$$
\min \operatorname{gr} C F^{\delta}\left(Y_{n(k+1)}(K)\right)-\max \operatorname{gr} C F^{\delta}\left(Y_{n}(K)\right) \leq \frac{c_{1}(\mathfrak{s})^{2}+n k}{4} .
$$

Clearly, if this inequality is not satisfied, then the $\operatorname{Spin}^{c}$ structure $\mathfrak{s}$ induces a trivial map from $C F^{\delta}\left(Y_{n}(K)\right)$ to $C F^{\delta}\left(Y_{n(k+1)}(K)\right)$. Thus, in view of Lemma 4.7, when $k$ is sufficiently large, the map

$$
\Pi_{s}^{A} \circ f_{1}^{\delta}: C F^{\delta}\left(Y_{n}(K)\right) \longrightarrow C F^{\delta}\left(Y_{n(k+1)}(K), s\right)
$$


is homogeneous, and hence there is an induced grading on $C F^{\delta}\left(Y_{n(k+1)}(K)\right)$ for which $f_{1}^{\delta}$ is a degree zero map. This induced grading, of course, depends on the intersection form on $X(k)$.

In the proof of Proposition 4.5, the mapping cone of $f_{2}^{\delta}$ is identified with the mapping cone of

$$
D_{n, i ; b}^{\delta}: \underset{\left\{\left.s \in \mathbb{Z}\right|_{s \equiv i}\right.}{\bigoplus} A_{s}^{\delta} \longrightarrow \underset{\{(\bmod n),-b \leq s \leq b\}}{\longrightarrow} \underset{\left\{\left.\in \mathbb{Z}\right|_{s \equiv i}\right.}{\bigoplus}{ }_{(\bmod n),-b+n \leq s \leq b\}}^{\delta},
$$

gotten by adding the maps

$$
v_{s}^{\delta}: A_{s}^{\delta} \longrightarrow B_{s}^{\delta} \text { and } h_{s}^{\delta}: A_{s}^{\delta} \longrightarrow B_{s+n}^{\delta}
$$

in the given range. Having just endowed $C F^{\delta}\left(Y_{n(k+1)}(K), s\right) \cong A_{s}^{\delta}$ with an absolute grading, there are absolute gradings on $B_{s}^{\delta}$ for which $v_{s}^{\delta}$ have degree zero. We claim that for these choices of absolute gradings, the map $h_{s}^{\delta}$ is also a grading-preserving map for $|s| \leq b$. This amounts to establishing that $v_{s}^{\delta} \circ \Pi_{s}^{A} \circ f_{1}^{\delta}$ and $h_{s+n}^{\delta} \circ \Pi_{s+n}^{A} \circ f_{1}^{\delta}$ are both homogeneous maps with the same degree (for brevity, we suppress here the identification of $A_{s}^{\delta} \cong C F^{\delta}\left(Y_{n(k+1)}, s\right)$ from the notation). Note first that both these maps are homogeneous, and that their degree difference is some constant $c$ depending on $i, s, n$, and $k$ (but independent of the knot $K$ ). Thus, to verify that $c=0$, it suffices to verify that $c=0$ in a single model case. More precisely, we need a particular $K \subset Y$ and chains $\xi \in C F^{+}\left(Y_{n}(K), i\right)$ so that for all sufficiently large $k, v_{s}^{+} \circ \Pi_{s}^{A} \circ f_{1}^{\delta}(\xi)$ and $h_{s+n}^{+} \circ \Pi_{s+n}^{A} \circ f_{1}^{\delta}(\xi)$ are homogeneous elements of the same degree.

To this end, we consider the unknot in $S^{3}$. Although $\Pi_{s}^{A} \circ f_{1}^{\delta}$ is, in general, difficult to calculate, in the case of the unknot, it is straightforward.

Specifically, fix any $\delta \geq 0$ and any $b \geq 0$. According to Proposition 4.5, for all sufficiently large $k$, the map $\phi^{\delta}=\left(f_{1}^{\delta}, H_{1}^{\delta}\right)$ induces a quasi-isomorphism

$$
C F^{\delta}\left(S^{3}, i\right) \longrightarrow \mathbb{X}_{i}^{\delta}(n ; b) .
$$

It follows from Section 2.6 that the induced map on homology must agree (up to an overall sign) with the map ८ from (5) or, more precisely, the restriction of $\iota$ to $H F^{\delta}\left(S^{3}\right)$. By taking $\delta$ sufficiently large, the map $\iota$ has non-trivial components in both $H_{*}\left(A_{s}^{\delta}\right)$ and $H_{*}\left(A_{s+n}^{\delta}\right)$ Moreover, provided $s>0$, the map $v_{s}^{\delta}$ induces an isomorphism in homology, and hence there is some cycle $\xi$ representing a non-trivial homology class (of arbitrary even degree). Since $\xi$ is a cycle, so is its image under the quasi-isomorphism with $\mathbb{X}_{i}^{\delta}(n ; b)$. In particular, for any integral $s$, we can choose $\delta$ sufficiently large for $\Pi_{s}^{A} \circ f_{1}^{\delta}(\xi)$ to be non-trivial, and even for its image under $v_{s}^{\delta}$ to be a non-trivial cycle in $B_{s}^{\delta}$. Since $f_{1}^{\delta}(\xi)$ is a cycle in the mapping cone, it follows that $h_{s-n}^{+} \circ \Pi_{s-n}^{A} \circ f_{1}^{\delta}(\xi)$ 
is homologous to $v_{s}^{+} \circ \Pi_{s}^{A} \circ f_{1}^{\delta}(\xi)$, and in particular, they are supported in the same degree.

\subsection{Completion of the proof of Theorem 4.1 in the case where $n>0$}

According to Proposition 4.6, an absolute grading on $C F^{\delta}\left(Y_{n}\right)$ induces absolute gradings on both

$$
\bigoplus_{-b \leq s \leq b} C F^{\delta}\left(Y_{n(k+1)}, s\right)
$$

and

$$
\bigoplus_{-b+n \leq s \leq b} T^{s} \otimes C F^{\delta}(Y) \subset C F^{\delta}(Y ; \mathbb{Z}[\mathbb{Z} / n k \mathbb{Z}])
$$

so that the maps $f_{1}^{\delta}$ and $f_{2}^{\delta}$ both are graded maps with degree zero. Let

$$
\Pi_{s}^{B}: C F^{\delta}(Y ; \mathbb{Z}[\mathbb{Z} / n k \mathbb{Z}]) \longrightarrow C F^{\delta}(Y) \cong B_{s}^{\delta}
$$

denote projection onto the summand of the form $T^{s} \otimes C F^{\delta}(Y)$.

Lemma 4.8 With respect to the above gradings, given $\delta \geq 0$, we have that for any $k$ sufficiently large and $|s| \leq b$, the map

$$
\Pi_{s}^{B} \circ H_{1}^{\delta}: C F^{\delta}\left(Y_{n}\right) \longrightarrow B_{s}^{\delta} \cong C F^{\delta}(Y)
$$

is a homogeneous map of degree +1 .

Proof $H_{1}^{\delta}$ splits as a sum of homotopy classes of maps of holomorphic quadrilaterals. These homotopy classes in turn correspond to $\operatorname{Spin}^{c}$ structures over the cobordism from $Y_{n}$ to $Y$. Since the intersection form on the composite cobordism $X(k) \cup_{Y_{n(k+1)}} W^{\prime}(k)$ is negative-definite, it follows that, $H_{1}^{\delta}$ is a sum of homogeneous maps, whose top order part corresponds to $\operatorname{Spin}^{c}$ structures over the composite cobordism whose first Chern classes have maximal square.

If the $\mathfrak{s}$ component of $H_{1}^{\delta}$ is non-trivial, then we claim that $\mathfrak{s} \mid X(k)$ is a maximal $\operatorname{Spin}^{c}$ structure over $X(k)$ in the sense that it appears in a component of $f_{1}^{\delta}$; and also, $\mathfrak{s} \mid W^{\prime}(k)$ is one of the top two $\operatorname{Spin}^{c}$ structures over $W^{\prime}(k)$ in the sense that it appears as a component of $f_{2}^{\delta}$.

To this end, note that with respect to the usual gradings on $C F^{+}\left(Y_{n}\right)$ and $C F^{+}(Y$; $\mathbb{Z}[\mathbb{Z} / n k \mathbb{Z}])$, the component of $H_{1}^{\delta}$ using the $\operatorname{Spin}^{c}$ structure $\mathfrak{s}$ is a homogeneous map of degree

$$
1+\frac{c_{1}(\mathfrak{s} \mid X(k))^{2}+n k}{4}+\frac{c_{1}\left(\mathfrak{s} \mid W^{\prime}(k)\right)^{2}+1}{4}
$$


Thus, this map is non-trivial only if

$$
\min \operatorname{grC} F^{+}(Y)-\max \operatorname{gr} C F^{+}\left(Y_{n}\right) \leq 1+\frac{c_{1}(\mathfrak{s} \mid X(k))^{2}+n k}{4}+\frac{c_{1}\left(\mathfrak{s} \mid W^{\prime}(k)\right)^{2}+1}{4}
$$

However,

$$
1+\frac{c_{1}(\mathfrak{s} \mid X(k))^{2}+n k}{4}+\frac{c_{1}\left(\mathfrak{s} \mid W^{\prime}(k)\right)^{2}+1}{4} \leq 1+\frac{c_{1}(\mathfrak{s} \mid X(k))^{2}+n k}{4}+\frac{1}{4},
$$

and since the left-hand-side of (23) is independent of $k$, it follows from Lemma 4.7 that (provided that $k$ is sufficiently large) $\mathfrak{s} \mid X(k)$ is one of the distinguished $\operatorname{Spin}^{c}$ structures on $X(k)$ satisfying inequality (22).

Similarly,

$$
1+\frac{c_{1}(\mathfrak{s} \mid X(k))^{2}+n k}{4}+\frac{c_{1}\left(\mathfrak{s} \mid W^{\prime}(k)\right)^{2}+1}{4} \leq 1+\frac{n k}{4}+\frac{c_{1}\left(\mathfrak{s} \mid W^{\prime}(k)\right)^{2}+1}{4} .
$$

For $k$ sufficiently large, this means also that $\mathfrak{s} \mid W^{\prime}(k)$ is one of the $\operatorname{Spin}^{c}$ structures satisfying (16); for if it were not, then by (17),

$$
1+\frac{n k}{4}+\frac{c_{1}\left(\mathfrak{s} \mid W^{\prime}(k)\right)^{2}+1}{4} \leq \frac{5-4 n}{4}-\frac{3 n k}{4},
$$

which, if $k$ is sufficiently large, violates (23).

We have thus verified that if the $\mathfrak{s}$-component of $H_{1}^{\delta}$ is non-trivial, then the restriction of $\mathfrak{s}$ to $X(k)$ and $W(n(k+1))$ are the ones which induce the map $\Pi_{s}^{A} \circ f_{1}^{\delta}$ and $\left.f_{2}^{\delta}\right|_{A_{s}^{\delta}}$ (with $|s| \leq b$ ) respectively.

Thus, it follows that $H_{1}^{\delta}$ is a homogeneous map from $C F^{+}\left(Y_{n}(K)\right)$ to $C F^{+}(Y$; $\mathbb{Z}[\mathbb{Z} / n k \mathbb{Z}]$ ) with respect to the "new gradings" (from Proposition 4.6) with degree +1 .

Proof of Theorem 4.1 for $n>0$ We endow the mapping cone $M\left(D_{n}^{\delta}\right)$ with an absolute grading where $\mathbb{A}^{\delta}$ has the inherited absolute grading, while $\mathbb{B}^{\delta}$ has the inherited grading, shifted up by one (this is done so that the differential on the mapping cone is a graded map which shifts grading down by -1$)$.

According to Proposition 4.5, the chain complex $C F^{\delta}\left(Y_{n}, i\right)$ is quasi-isomorphic to the mapping cone $\mathbb{X}_{i}^{\delta}(n ; b)=M\left(D_{n, i ; b}^{\delta}\right)$ via a quasi-isomorphism induced by $\phi^{\delta}=\left(f_{1}^{\delta}, H_{1}^{\delta}\right)$ which, according to the above lemma, is a grading-preserving map.

In view of Lemma 4.3, this gives, for any $\delta \geq 0$ a grading-preserving isomorphism of $H F^{\delta}\left(Y_{n}, i\right)$ with $H_{*}\left(\mathbb{X}_{i}^{\delta}(n)\right)$. It follows from Lemma 2.7 that in fact $H F^{+}\left(Y_{n}, i\right) \cong$ $H_{*}\left(\mathbb{X}_{i}^{+}(n)\right)$. 


\subsection{Negative integral surgeries}

In fact, the above proof of Theorem 4.1 for positive surgery coefficients adapts with minor changes to the case of negative surgery coefficients. We outline the changes presently.

Proof of Theorem 4.1 for negative surgery coefficients We will continue to assume $n>0$, only now, we shall consider the three-manifold $Y_{-n}(K)$.

We start by considering the changes to Section 4.3. In the present case, we apply Theorem 3.1, only now focusing on the maps

$$
C F^{+}\left(Y_{n(k-1)}\right) \stackrel{f_{2}^{+}}{\longrightarrow} C F^{+}(Y ; \mathbb{Z}[\mathbb{Z} / n k \mathbb{Z}]) \stackrel{f_{3}^{+}}{\longrightarrow} C F^{+}\left(Y_{-n}(K)\right),
$$

where again we take $k$ to be sufficiently large.

In place of (21), we have

$$
\mathfrak{m}\left(\mathfrak{x}_{s}\right)-\mathfrak{m}\left(\mathfrak{y}_{s}\right)=-n(k-1) \equiv n \quad(\bmod n k) .
$$

Thus, it follows that, unlike the conventions specified in (21), the components of $f_{1}^{+}$ corresponding to $\mathfrak{x}_{s}$ and $\mathfrak{y}_{s}$ are maps

$$
\widehat{\mathfrak{v}}_{s}: \widehat{\mathfrak{A}}_{s} \longrightarrow \widehat{\mathfrak{B}}_{s} \text { and } \quad \widehat{\mathfrak{h}}_{s}: \widehat{\mathfrak{A}}_{s} \longrightarrow \widehat{\mathfrak{B}}_{s-n} .
$$

Proceeding as in Section 4.3, for all sufficiently large $k$, we identify the mapping cone of $f_{2}^{+}$with the mapping cone of

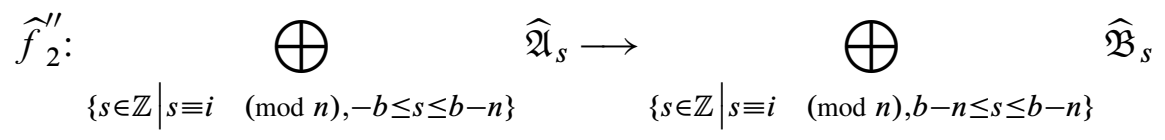

This is then identified with the mapping cone $\widehat{\mathbb{X}}_{i}(-n ; b)$, via Theorem 2.3 as before.

Indeed, the modifications from Section 4.4 carry over, to prove the analogue of Proposition 4.5 , using surgery coefficient $-n$.

When studying gradings ( $\mathrm{cf}$ Section 4.5), now, we study the map $f_{3}^{+}$belonging to the cobordism $W_{-n}(K)$, rather than the map $f_{1}^{+}$, since now $W_{-n}(K)$ is a negative-definite cobordism.

Of course, for fixed $\delta \geq 0$ and $k$ large enough, $f_{2}^{\delta}$ remains a homogeneous map according to Lemma 4.4, as before. Moreover, we claim that the same holds for $f_{3}^{\delta}$. To see this, consider the cobordism $W_{-n}(K): Y \longrightarrow Y_{-n}(K)$. Now, $(\operatorname{cf}(12)) f_{3}^{\delta}\left(\xi \otimes T^{i}\right)$ consists of a sum of maps associated to $\operatorname{Spin}^{c}$ structures $\mathfrak{s}$ which differ by addition of $k \cdot[\widehat{F}]$ (where here $\widehat{F}$ represents a generator for $H_{2}\left(W_{-n}(K)\right)$, and in particular $[\widehat{F}] \cdot[\widehat{F}]=-n)$. 
Lemma 4.9 Fix an integer $\delta \geq 0$ and a constant $C_{0}$. For all sufficiently large $k$, the following holds. For each $\mathfrak{s}_{0} \in \operatorname{Spin}^{c}\left(W_{-n}(K)\right)$, there is at most one $\operatorname{Spin}^{c}$ structure $\mathfrak{s} \in \mathfrak{s}_{0}+k \operatorname{PD}[\widehat{F}] \mathbb{Z}$ for which

$$
C_{0} \leq c_{1}(\mathfrak{s})^{2}+n k
$$

Proof Let $\mathfrak{s}$ be a $\operatorname{Spin}^{c}$ structure as above. In this case, $c_{1}(\mathfrak{s})=\alpha \cdot \operatorname{PD}[\widehat{F}]$, where $\alpha$ satisfies

$$
|\alpha| \leq \sqrt{\frac{n k-C_{0}}{n}} \leq 2 \sqrt{k}
$$

if $k$ is sufficiently large. But then, for any other $\operatorname{Spin}^{c}$ structure, of the form $\mathfrak{s}_{0}+$ $(k \ell) \operatorname{PD}[\widehat{F}]$ (with $\ell \in \mathbb{Z}$ ), we have that

$$
\begin{aligned}
c_{1}\left(\mathfrak{s}^{\prime}\right)^{2}-c_{1}(\mathfrak{s})^{2} & \leq 4\left(k\left\langle c_{1}(\mathfrak{s}),[\widehat{F}]\right\rangle-k^{2} n\right) \\
& \leq-4 k^{2} n\left(1-\frac{2}{\sqrt{k}}\right) \\
& \leq-n k^{2}
\end{aligned}
$$

if $k$ is sufficiently large.

Now, if

$$
f_{W_{-n}(K), \mathfrak{s}}^{\delta}: C F^{\delta}(Y) \longrightarrow C F^{\delta}\left(Y_{-n}(K)\right)
$$

is non-trivial, then according to (1),

$$
c_{1}(\mathfrak{s})^{2} \geq-1+4\left(\min \operatorname{gr} C F^{\delta}\left(Y_{-n}(K)\right)-\max \operatorname{gr} C F^{\delta}(Y)\right),
$$

so the above lemma shows that for large enough $k$, there is a unique $\operatorname{Spin}^{c}$ structure which contributes non-trivially to $f_{3}^{\delta}\left(\cdot \otimes T^{i}\right)$. (This is the analogue of Lemma 4.7.)

Thus, a grading on $C F^{+}\left(Y_{-n}(K)\right)$ induces gradings on $C F^{+}(Y ; \mathbb{Z}[\mathbb{Z} / n k \mathbb{Z}])$ and from there also on $C F^{+}\left(Y_{n(k-1)}\right)$ (note that $f_{2}^{\delta}$ remains a graded map for all sufficiently large $k$ by Lemma 4.4). To check that these gradings are compatible, now, we use the example of the unknot with framing $-n$.

Finally, it remains to show that quasi-isomorphism

$$
\psi^{\delta}: M\left(f_{2}^{\delta}\right) \longrightarrow C F^{+}\left(Y_{-n}(K)\right)
$$

defined by $\psi^{\delta}(x, y)=H_{2}^{\delta}(x)+f_{3}^{\delta}(y)$ is a relatively graded map. This follows from an analogue of Lemma 4.8 .

To this end, note that $H_{2}^{\delta}: C F^{\delta}\left(Y_{n(k-1)}\right) \longrightarrow C F^{\delta}\left(Y_{-n}\right)$ is defined as a count of quadrilaterals. Thus, if the map

$$
H_{2}^{\delta}: C F^{\delta}\left(Y_{n(k-1)}(K)\right) \longrightarrow C F^{\delta}\left(Y_{-n}(K)\right)
$$

Algebraic $8 \mathcal{G}$ Geometric Topology, Volume 8 (2008) 
is non-trivial, then

$$
\begin{aligned}
& \min \operatorname{grC} F^{+}\left(Y_{-n}(K)\right)-\max \operatorname{grC} F^{+}\left(Y_{n(k-1)}(K)\right) \\
& \leq 1+\frac{\left.c_{1}(\mathfrak{s} \mid W)\right)^{2}+c_{1}\left(\mathfrak{s} \mid W^{\prime \prime}(k)\right)^{2}+2}{4}
\end{aligned}
$$

where here $W^{\prime \prime}(k)$ indicates the cobordism $W_{n(k-1)}^{\prime}: Y_{n(k-1)} \longrightarrow Y$. But the lefthand-side is bounded below by constants (independent of $k$ ) plus $-n k / 4$ (cf Corollary 2.4). It follows now from Lemma 4.9 that $\mathfrak{s} \mid W^{\prime \prime}(k)$ is one of the distinguished $\operatorname{Spin}^{c}$ structures satisfying the inequality in Lemma 4.9; it also follows from Lemma 4.4 that $\mathfrak{s} \mid W(k)$ is one of the distinguished $\operatorname{Spin}^{c}$ structures satisfying (16). Thus, $H_{2}^{\delta}$ shifts grading up by -1 , and $\psi^{\delta}$ is a relatively graded map. This completes the proof of Theorem 4.1 for negative integer surgeries.

\subsection{The case where $n=0$}

We have not treated the case where $n=0$, as it follows more quickly from the existing exact sequences. Specifically, the integer surgeries long exact sequence (of [15, Theorem 10.19]) shows that $H F^{+}\left(Y_{0}(K), i\right)$ is identified with the homology of the mapping cone of

$$
v_{i}^{+}+h_{i}^{+}: A_{i}^{+} \longrightarrow B^{+} \text {. }
$$

Indeed, considering the integer surgeries exact sequence with twisted coefficients [15, Theorem 10.23], we see that $H_{*}\left(\mathbb{X}_{i}^{+}(0)\right)$ calculates $\underline{H F}^{+}\left(Y_{0}(K), i\right)$.

\subsection{Maps induced by cobordisms}

The mapping cone $\mathbb{X}^{+}(n)$ provides also a model for the maps induced by the cobordism from $Y$ to $Y_{n}(K)$, according to Theorem 4.2. The proof of this result follows quickly from our proof of Theorem 4.1.

Proof of Theorem 4.2 Observe that in Theorem 3.1 (in the notation of Section 3), the following square commutes:

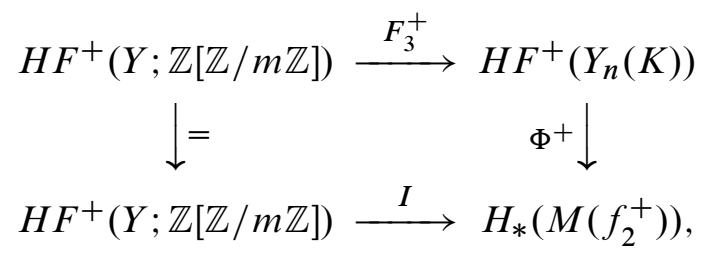

where here $\Phi^{+}$is the map in homology induced by the quasi-isomorphism $\phi^{+}$of (13), $I$ is the map on homology induced by the canonical map $\iota$ to the mapping cone, and $F_{3}^{+}$is the map on homology induced by the map $f_{3}^{+}$from (11). 
Let $m=|n| k$ with $k$ sufficiently large, as we have done above. Fix also $s \in \mathbb{Z}$ and $\delta \geq 0$. Although the restriction of

$$
f_{3}^{\delta}: C F^{\delta}(Y ; \mathbb{Z}[\mathbb{Z} / n k \mathbb{Z}]) \longrightarrow C F^{\delta}\left(Y_{n}(K)\right)
$$

to $T^{s} \otimes C F^{\delta}(Y) \subset C F^{\delta}(Y ; \mathbb{Z}[\mathbb{Z} / n k \mathbb{Z}])$ in principle is a sum of maps induced by Spin $^{c}$ structures over $W_{n}(K)$; all these are in the $k \operatorname{PD}[\widehat{F}] \cdot \mathbb{Z}$-orbit of a given one (cf (12)). Thus, for fixed $\delta$ and $k$ sufficiently large, it is easy to see that there is in each such orbit, a single chain map which could conceivably be non-trivial, and that is the map $F_{W, \mathfrak{s}}^{+}$induced by the $\operatorname{Spin}^{c}$ structure with $\left\langle c_{1}(\mathfrak{s}),[\widehat{F}]\right\rangle+n=2 s$.

Thus, we have the following commutative square:

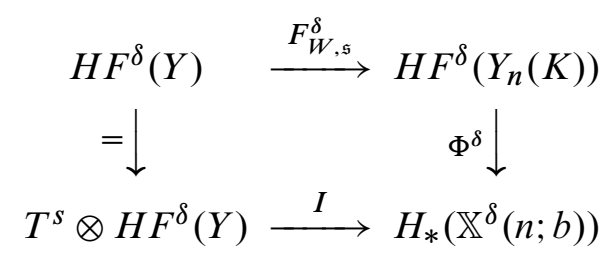

(here, $b$ is a truncation as usual, which now we choose so that $b>s$ ). Note that $\delta$ here is arbitrary, and all maps are graded. Thus, Lemma 2.7 provides the canonical extensions making the following square commute:

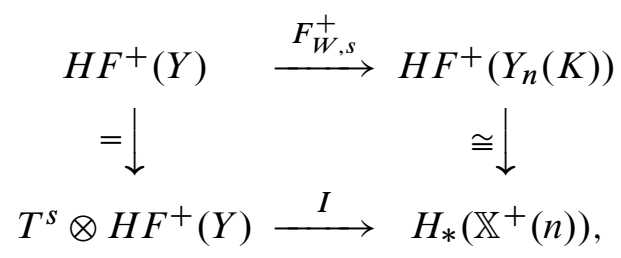

proving the theorem.

\subsection{Generalizations}

The proof of Theorem 1.1 applies immediately in any context where $Y$ has a relatively $\mathbb{Z}$-graded Floer homology.

Specifically, let $Y$ be a closed, oriented three-manifold, and fix a $\operatorname{Spin}^{c}$ structure $\mathfrak{t}$ over $Y$ with torsion first Chern class. Let $K \subset Y$ be a null-homologous knot in $Y$, and fix a Seifert surface $F \subset Y$ for $K$.

In this case, the space of $\operatorname{Spin}^{c}$ structures over $Y_{n}(K)$ which are $\operatorname{Spin}^{c}$-cobordant to $\mathfrak{t}$ over the cobordism $W_{n}^{\prime}(K)$ is identified with $\mathbb{Z} / n \mathbb{Z}$, as in Lemma 2.2. For $i \in \mathbb{Z} / n \mathbb{Z}$, let $C F^{+}\left(Y_{n}(K), i, \mathfrak{t}\right)$ denote the corresponding summand of $C F^{+}\left(Y_{n}(K)\right)$. In this 
case, we let $A_{s, \mathfrak{t}}^{+}$denote the corresponding chain complex associated to the knot filtration on $C F^{+}(Y, \mathfrak{t})$, and let $B_{s, \mathfrak{t}}^{+}$denote $C F^{+}(Y, \mathfrak{t})$. Write

$$
\mathbb{A}_{i, \mathrm{t}}^{+}=\bigoplus_{\left\{\left.s \in \mathbb{Z}\right|_{s \equiv i}(\bmod n)\right\}} A_{s, \mathrm{t}}^{+} \text {and } \quad \mathbb{B}_{i, \mathrm{t}}^{+}=\bigoplus_{\{s \in \mathbb{Z} \mid s \equiv i(\bmod n)\}} B_{s, \mathrm{t}}^{+}
$$

Theorem 4.10 Fix a Spin ${ }^{c}$ structure $\mathfrak{t}$ over $Y$ whose first Chern class is torsion, and a null-homologous knot $K \subset Y$. For each $i \in \mathbb{Z} / n \mathbb{Z}$, the mapping cone $\mathbb{X}_{i, t}^{+}(n)$ of

$$
D_{n, i, \mathfrak{t}}^{+}: \mathbb{A}_{i, \mathfrak{t}}^{+} \longrightarrow \mathbb{B}_{i, \mathfrak{t}}^{+}
$$

is identified with $C F^{+}\left(Y_{n}(K), i, \mathfrak{t}\right)$ as graded $\mathbb{Z}[U]$-modules.

Of course, when $n= \pm 1, \operatorname{Spin}^{c}\left(Y_{n}(K)\right) \cong \operatorname{Spin}^{c}(Y)$, and there is no additional choice of $i \in \mathbb{Z} / n \mathbb{Z}$. In this case, we write simply $\mathbb{X}_{\mathfrak{t}}^{+}(n)$ for the mapping cone.

The theorem follows at once from the methods of this section: specifically, our proof of Theorem 4.1 uses the fact that $Y$ is an integer homology three-sphere only in that $C F^{+}(Y)$ has a relative $\mathbb{Z}$-grading.

\section{Sample calculations}

We give some calculations to illustrate the techniques of this paper. In Section 5.1, we use Theorem 4.1 to calculate the Heegaard Floer homology groups of \pm 1 -surgeries on the torus knot $T_{3,4}$ in the three-sphere. (Of course, \pm 1 surgeries on torus knots are Brieskorn spheres, and as such their Heegaard Floer homology groups can be calculated using the techniques of Ozsváth-Szabó [13]; however, we find it instructive to sketch here the calculations using present methods.) In Section 5.2, we include a calculation of the Heegaard Floer homology of a non-trivial circle bundle over a closed, oriented two-manifold.

\subsection{Surgeries on the torus knot $T_{3,4}$}

Sufficiently large surgeries on torus knots have particularly simple Heegaard Floer homology groups - in each $\operatorname{Spin}^{c}$ structure, $H F^{+}\left(S_{N}^{3}(K)\right) \cong \mathcal{T}^{+}$, ie these spaces are "Heegaard Floer homology lens spaces", or $L$-spaces in the terminology from [18]. As such, the filtered homotopy type of the knot filtration of torus knots is immediately determined by the Alexander polynomial, cf [18, Theorem 1.2]. 
For example, that result shows that the filtered chain complex $C$ for the torus knot $K=T_{3,4}$ has five generators as a $\mathbb{Z}\left[U, U^{-1}\right]$-module, $\left\{x_{i}\right\}_{i=1}^{5}$, with

$$
\begin{array}{cc}
\mathcal{F}\left(x_{1}\right)=(0,3) & \operatorname{gr}\left(x_{1}\right)=0 \\
\mathcal{F}\left(x_{2}\right)=(0,2) & \operatorname{gr}\left(x_{2}\right)=-1 \\
\mathcal{F}\left(x_{3}\right)=(0,0) & \operatorname{gr}\left(x_{3}\right)=-2 \\
\mathcal{F}\left(x_{4}\right)=(0,-2) & \operatorname{gr}\left(x_{4}\right)=-5 \\
\mathcal{F}\left(x_{5}\right)=(0,-3) & \operatorname{gr}\left(x_{5}\right)=-6 .
\end{array}
$$

Moreover, the non-trivial differentials are

$$
\partial x_{2}=U \cdot x_{1}+x_{3} \quad \text { and } \quad \partial x_{4}=U^{2} \cdot x_{3}+x_{5} .
$$

The filtrations and the differentials are illustrated in Figure 2.

From this, we quickly see the following Lemma.

Lemma 5.1 $H_{*}\left(A_{s}^{+}\right) \cong H_{*}\left(B_{s}^{+}\right) \cong \mathcal{T}^{+}$. Moreover, under this identification, the maps on homology induced by

$$
h_{s}^{+}: A_{s}^{+} \longrightarrow B_{s+n}^{+} \text {and } v_{-s}^{+}: A_{-s}^{+} \longrightarrow B_{-s}^{+}
$$

are identified with multiplication by

$$
\begin{cases}U^{3} & \text { if } s=2 \\ U^{2} & \text { if } s=1 \\ U & \text { if } s=0,-1,-2 \\ 1 & \text { if } s<-2\end{cases}
$$

Proof From the above description of $C$, we see that $A_{s}^{+}=B_{s}^{+}$and $A_{-s}^{+}=B_{-s-1}^{+}$ whenever $s>2$. It remains to consider the remaining cases with $|s| \leq 2$. For example, $A_{1}^{+}$contains three additional generators not contained in $B_{1}^{+}: U x_{1}, U^{2} x_{1}$, and $U x_{2}$. However, these latter two generators do not contribute to $H_{*}\left(A_{1}^{+}\right): \partial U x_{2}=U^{2} x_{1}$ (in $A_{1}^{+}$). The bottom-most non-trivial homology class in $H_{*}\left(A_{1}^{+}\right)$is represented by any of the three cycles $U x_{1}, x_{3}$, or $U^{-2} x_{5}$. However, these cycles are boundaries in $B_{1}^{+}$. It follows quickly that $v_{1}^{+}: \mathcal{T}^{+} \cong H_{*}\left(A_{1}^{+}\right) \longrightarrow H_{*}\left(B_{1}^{+}\right) \cong \mathcal{T}^{+}$is modeled on multiplication by $U$.

The other cases follow similarly.

The chain complex for $\mathbb{X}^{+}(1)$ is summarized in Figure 3.

Algebraic $8 \mathcal{G}$ Geometric Topology, Volume 8 (2008) 


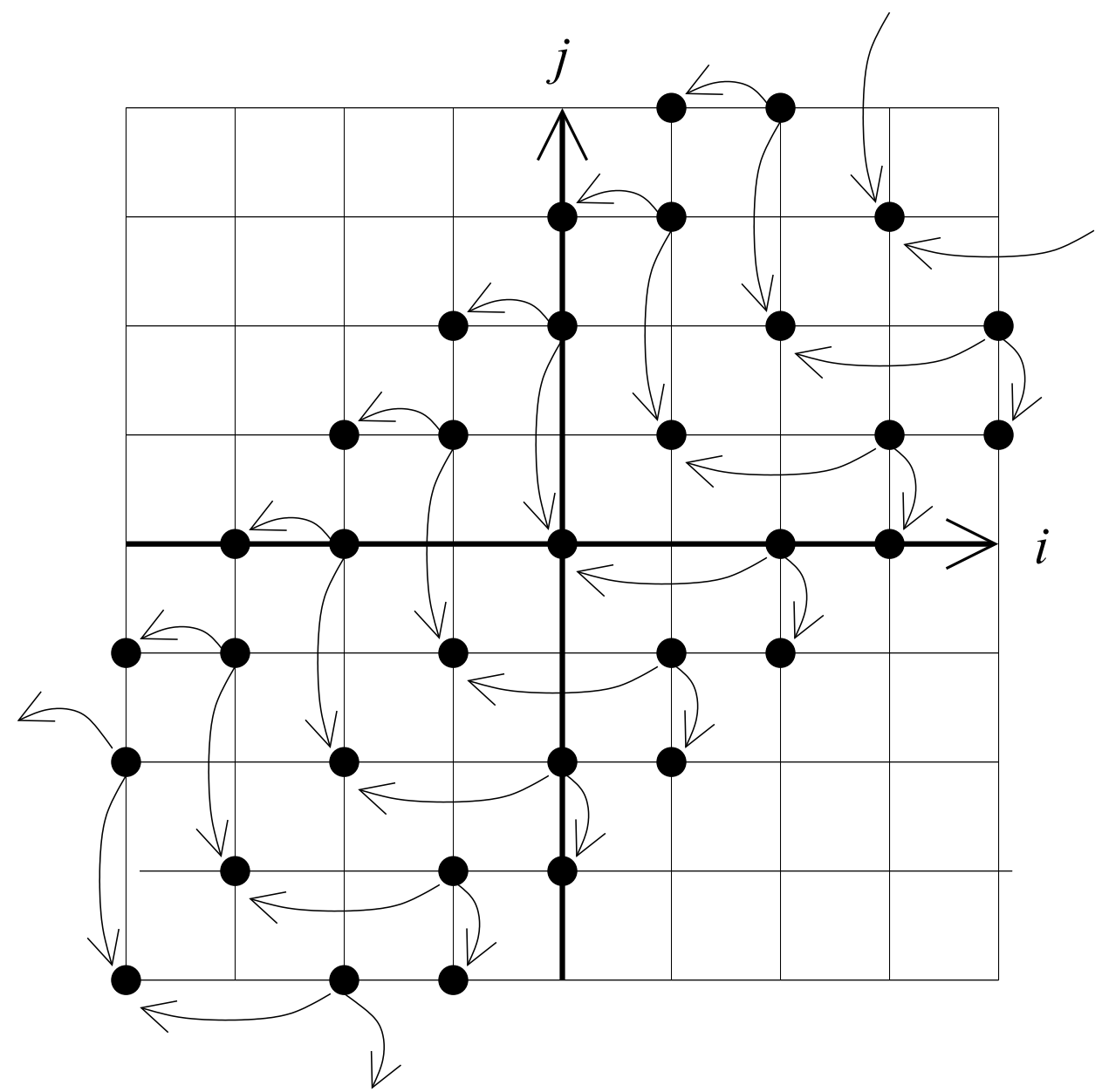

Figure 2: The doubly-filtered knot complex for the torus knot $T_{3,4}$. We have illustrated here the plane, representing filtration levels of generators. A dot at a lattice point $(i, j)$ represents a group $\mathbb{Z}$ with filtration $(i, j)$. The five generators of $C$ as a $\mathbb{Z}\left[U, U^{-1}\right]$-module are represented by the five dots on the vertical axis.

In view of Lemma 5.1, the map on homology $H_{*}\left(\mathbb{A}^{+}\right) \longrightarrow H_{*}\left(\mathbb{B}^{+}\right)$induced by $D^{+}$is surjective, and hence $H_{*}\left(\mathbb{X}^{+}(1)\right)$ can be identified with its kernel. Indeed, $H_{*}\left(\mathbb{X}^{+}(1)\right)$ consists of a submodule isomorphic to $A_{0}^{+}$, and also four additional generators represented by $1 \in \mathcal{T}^{+} \cong H_{*}\left(A_{s}^{+}\right)$for $0<|s| \leq 2$. According to the grading conventions, the two bottom-most generators of $A_{ \pm 1}^{+}$are supported in degree -2 and the two in $A_{ \pm 2}^{+}$are supported in degree 0 . Thus, in light of Theorem 4.1, these 


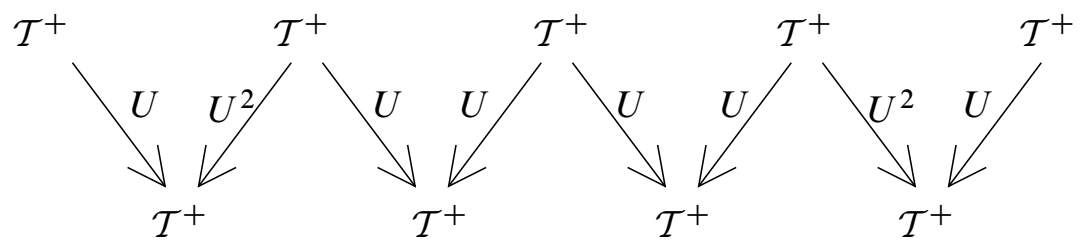

Figure 3: Chain complex for $S_{+1}^{3}\left(T_{3,4}\right)$. The results of Lemma 5.1 can be summarized by this diagram, which represents the chain complex $\mathbb{X}^{+}(1)$. (Note that we have illustrated here a truncated portion; which carries all of the homology of $\mathbb{X}^{+}(1)$.) The arrows represent the differential. This diagram contains information about gradings as well: the relative grading is characterized by the requirement that the differential drops grading by one.

calculations show that:

$$
H F^{+}\left(S_{1}^{3}\left(T_{3,4}\right)\right) \cong \mathcal{T}_{-2}^{+} \oplus \mathbb{Z}_{(-2)}^{2} \oplus \mathbb{Z}_{(0)}^{2} .
$$

Here, $\mathcal{T}_{d}^{+}$denotes a copy of $\mathcal{T}^{+}$, thought of as a graded $\mathbb{Z}[U]$-module, graded so that its element of lowest degree is supported in degree $d$; while $\mathbb{Z}_{(d)}$ denotes $\mathbb{Z} \cong \mathbb{Z}[U] / U$, supported in degree $d$.

For $(-1)$-surgery on $T_{3,4}$, Lemma 5.1 gives rise to the chain complex for $\mathbb{X}^{+}(-1)$ illustrated in Figure 4.

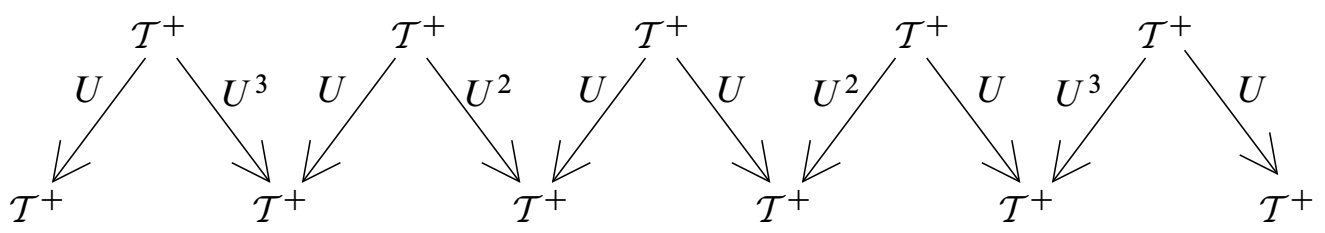

Figure 4: Chain complex for $S_{-1}^{3}\left(T_{3,4}\right)$. This is an illustration of the complex $\mathbb{X}^{+}(-1)$, with the same conventions as in Figure 3 .

In this case, we can calculate:

$$
H F^{+}\left(S_{-1}^{3}\left(T_{3,4}\right)\right) \cong \mathcal{T}_{0}^{+} \oplus \mathbb{Z}_{(-1)} \oplus \mathbb{Z}_{(-3)}^{2} \oplus \mathbb{Z}_{(-7)}^{2}
$$

(These can be compared with the calculations from [13], bearing in mind that $S_{+1}^{3}\left(T_{3,4}\right) \cong-\Sigma(3,4,11)$ and $S_{-1}^{3}\left(T_{3,4}\right) \cong \Sigma(3,4,13)$, where $\Sigma(p, q, r)$ denotes the Brieskorn sphere with multiplicities $p, q$, and $r$, given its orientation as the boundary of a negative-definite plumbing.)

Algebraic ${ }^{3} \mathcal{G}$ Geometric Topology, Volume 8 (2008) 


\subsection{Non-trivial circle bundles over Riemann surfaces}

Recall that for any closed, oriented three-manifold, the groups $U^{d} \cdot H F^{+}(Y) \subset$ $H F^{+}(Y)$ stabilize for sufficiently large $d$, and hence we can define the "reduced Floer homology group" $H F_{\text {red }}^{+}(Y)=H F^{+}(Y) / U^{d} \cdot H F^{+}(Y)$ for all sufficiently large $d$. As an illustration of Theorem 1.1, or more precisely, its generalization in Theorem 4.10, we calculate the reduced Floer homology of a non-trivial circle bundle over a Riemann surface of genus $g$, with coefficients in $\mathbb{Z} / 2 \mathbb{Z}$.

To explain how this is done, recall that there is a genus $g$ fibered knot in $\#^{2 g}\left(S^{2} \times S^{1}\right)$, the "Borromean knot", constructed as follows. Consider the Borromean link, with a distinguished component. Form the $g$-fold connected sum of the Borromean link, where the connected sum is performed along the distinguished components. Then, form zero-surgeries on all the remaining components, viewing the distinguished component as a knot inside $\#^{2 g}\left(S^{2} \times S^{1}\right)$. The three-manifold which is an Euler number $n$ circle bundle over the genus $g$ Riemann surface is obtained from $n$-framed surgery on this knot.

The knot Floer complex for the Borromean knot is calculated in [14, Proposition 9.2]. It is proved there that $C \cong \Lambda^{*} H^{1}(\Sigma ; \mathbb{Z}) \otimes \mathbb{Z}\left[U, U^{-1}\right]$. The $\mathbb{Z} \oplus \mathbb{Z}$ filtration is given by

$$
C\{i, j\}=U^{-i} \otimes \Lambda^{g-i+j} H^{1}(\Sigma ; \mathbb{Z}),
$$

and the entire group $C\{i, j\}$ is supported in dimension $i+j$. All the differentials on this knot complex are trivial, and indeed, so are all the higher differentials; ie

$$
H F^{\infty}\left(\#^{2 g}\left(S^{2} \times S^{1}\right)\right) \cong \bigoplus_{i, j} C\{i, j\}
$$

Indeed, these groups have some additional structure which can be identified. Specifically, recall (cf [16, Subsection 4.2.5]) that if $Y$ is an oriented three-manifold, then there is an action of $\Lambda^{*} H_{1}(Y ; \mathbb{Z}) /$ Tors on all the versions of Floer homology. For the case where $Y \cong \#^{2 g}\left(S^{2} \times S^{1}\right)$, under the identification $H_{1}(\Sigma ; \mathbb{Z}) \cong H_{1}\left(\#^{2 g}\left(S^{2} \times S^{1}\right)\right)$ and $H F^{\infty}\left(\#^{2 g}\left(S^{2} \times S^{1}\right)\right) \cong \Lambda^{*} H_{1}(\Sigma ; \mathbb{Z}) \otimes_{\mathbb{Z}} \mathbb{Z}\left[U, U^{-1}\right]$ described above, the action of $\gamma \in H_{1}(\Sigma ; \mathbb{Z})$ is given by the formula

$$
\gamma \cdot\left(\omega \otimes U^{j}\right)=\left(\iota_{\gamma} \omega\right) \otimes U^{j}+\operatorname{PD}(\gamma) \wedge \omega \otimes U^{j+1},
$$

where here $\iota_{\gamma}$ denotes contraction (cf the proof of [14, Theorem 9.3]).

It is significantly easier to work with coefficients in $\mathbb{F}=\mathbb{Z} / 2 \mathbb{Z}$, because in that case, the induced homotopy equivalence between $C\{i \geq 0\}$ and $C\{j \geq 0\}$ takes a particularly 
simple form. Write $\bar{C}$ for $C \otimes \mathbb{F}$ (and writing, for example, $\bar{C}\{i, j\}$ for $C\{i, j\} \otimes \mathbb{F}$. In this case, we have the following Proposition.

Proposition 5.2 Consider the natural homotopy equivalence

$$
\phi: C\{i \geq 0\} \longrightarrow C\{j \geq 0\} .
$$

Its induced map $\bar{\phi}: \bar{C}\{i \geq 0\} \longrightarrow \bar{C}\{j \geq 0\}$ sends $\bar{C}\{i, j\}$ to $\bar{C}\{j, i\}$.

The above proposition does not hold over $\mathbb{Z}$. A description of the involution over $\mathbb{Z}$ is given in Jabuka-Mark [3].

In fact we will be able to calculate the homotopy equivalence $\bar{\phi}$, but first, we need a lemma.

Lemma 5.3 Consider the graded module

$$
M=\Lambda^{*} H^{1}(\Sigma ; \mathbb{Z}) \otimes \mathbb{Z}\left[U, U^{-1}\right] / \mathbb{Z}[U]
$$

over the ring $\Lambda^{*} H_{1}(\Sigma ; \mathbb{Z}) \otimes_{\mathbb{Z}} \mathbb{Z}[U]$ with the action defined in (25). The only automorphisms of $M$ are multiplication by \pm 1 . Similarly, if we consider $\bar{M}=M \otimes \mathbb{F}$ over the ring $\Lambda^{*} H_{1}(\Sigma ; \mathbb{Z}) \otimes_{\mathbb{Z}} \mathbb{F}[U]$, where $\mathbb{F}=\mathbb{Z} / 2 \mathbb{Z}$, the only automorphism of $\bar{M}$ is the identity map.

Proof Let $\phi$ be an automorphism of $M$ as a module over $\Lambda^{*} H_{1}(\Sigma ; \mathbb{Z}) \otimes_{\mathbb{Z}} \mathbb{Z}[U]$. Consider the filtration of $M$ by the submodules $\left\{M_{i}\right\}_{i=1}^{\infty}$ where $M_{i}$ is the set of elements annihilated by $U^{i}$. Clearly, these submodules exhaust $M$, and $\phi_{i}$ preserves this filtration.

Consider the summand $\mathbb{Z} \cong \Lambda^{2 g} H^{1}(\Sigma ; \mathbb{Z}) \otimes U^{-i} \subset M_{i}$. This consists of those elements of $M_{i}$ with maximal grading, and hence it is preserved by $\phi$. Thus, $\phi$ must induce multiplication by \pm 1 on this summand. However, this summand generates all of $M_{i}$ as a module over $\Lambda^{*} H_{1}(\Sigma ; \mathbb{Z}) \otimes \mathbb{Z}[U]$. It follows at once that $\phi$ induces multiplication by \pm 1 on all of $M_{i}$.

The same argument shows that an automorphism $\bar{\phi}$ of $\bar{M}$ is the identity map.

Proof of Proposition 5.2 We describe the homotopy equivalence $\bar{\phi}$ explicitly (up to an overall sign). For a fixed orientation on $\Sigma$, let $\left\{A_{i}, B_{i}\right\}_{i=1}^{g}$ be a symplectic basis of homology classes; ie \# $\left(A_{i} \cap B_{j}\right)=\delta_{i, j}$ (Kronecker $\delta$ ), while \# $\left(A_{i} \cap A_{j}\right)=$ $\#\left(B_{i} \cap B_{j}\right)=0$ for all $i, j$. There is an induced map

$$
I: \Lambda^{k} H^{1}(\Sigma ; \mathbb{Z}) \longrightarrow \Lambda^{k} H^{1}(\Sigma ; \mathbb{Z}),
$$


which commutes with wedge product and satisfies $I\left(A_{i}^{*}\right)=-B_{i}^{*}$ and $I\left(B_{i}^{*}\right)=A_{i}^{*}$. Also, there is a Hodge star operator

$$
*: \Lambda^{k} H^{1}(\Sigma ; \mathbb{Z}) \longrightarrow \Lambda^{2 g-k} H^{1}(\Sigma ; \mathbb{Z})
$$

belonging to a metric where $A_{i}$ and $B_{i}$ have length one.

Consider the map

$$
\phi_{0}: C\{i, j\} \longrightarrow C\{j, i\}
$$

induced by

$$
\omega \otimes U^{-i} \mapsto(* I \omega) \otimes U^{-j} .
$$

It is easy to see that $\phi_{0}$ extends to an isomorphism of $\mathbb{Z}$-modules, which commutes up to sign with the action by $U$ and $\Lambda^{*} H_{1}(\Sigma ; \mathbb{Z})$.

As such, it induces a graded $\Lambda^{*} H_{1}(\Sigma ; \mathbb{Z}) \otimes_{\mathbb{Z}} \mathbb{F}[U]$-equivariant isomorphism from $\bar{C}\{i \geq 0\}$ to $\bar{C}\{j \geq 0\}$. It follows from Lemma 5.3 that this map is the only $\Lambda^{*} H_{1}(\Sigma ; \mathbb{Z}) \otimes \mathbb{F}[U]$-equivariant isomorphism from $\bar{C}\{i \geq 0\}$ to $\bar{C}\{j \geq 0\}$.

It will be useful to have the following terminology in place before stating our results.

Definition 5.4 Let $M$ be a module over $\mathbb{F}[U]$, and $m \in M$. The length of $m, L_{M}(m)$, is the largest integer $\ell$ with the property that $U^{\ell} \cdot m \neq 0$.

Note that if $m \in \bar{C}\{i, j\} \subset A_{s}^{+}$, then

$$
L_{A_{s}^{+}}(m)=\max (i, j-s) .
$$

Let $X(g, d)$ be the module over $\mathbb{Z}[U]$ given by

$$
X(g, d)=\bigoplus_{i=0}^{d} \Lambda^{2 g-i} H^{1}\left(\Sigma_{g}\right) \otimes_{\mathbb{Z}}\left(\mathbb{Z}[U] / U^{d-i+1}\right) .
$$

In fact, this is a module over the ring $\mathbb{Z}[U] \otimes_{\mathbb{Z}} \Lambda^{*} H_{1}\left(\Sigma_{g}\right)$, where the action of $\gamma \in H_{1}\left(\Sigma_{g}\right)$ is given by (25). We can endow $X(g, d)$ with a grading which is lowered by one by $D_{\gamma}$, lowered by two by multiplication with $U$, and centered at zero, in the sense that the summand $\Lambda^{2 g-d} H^{1}\left(\Sigma_{g}\right)$ is supported in degree zero. Note that if $\operatorname{Sym}^{d}\left(\Sigma_{g}\right)$ denotes the $d$-fold symmetric product of the surface $\Sigma_{g}$, then

$$
H_{*}\left(\operatorname{Sym}^{d}\left(\Sigma_{g}\right)\right) \cong X_{*-2 d}(g, d),
$$

cf Macdonald [8].

In order to apply Proposition 5.2, we will use coefficients in $\mathbb{F}=\mathbb{Z} / 2 \mathbb{Z}$. In the interest of clarity, we restrict attention here to the case where the Euler number $n=1$. The general case is addressed in Theorem 5.6 below.

Algebraic 83 Geometric Topology, Volume 8 (2008) 
Theorem 5.5 Let $Y$ be the circle bundle over a Riemann surface of genus $g$ with Euler number 1 . Let $\mathbb{F}=\mathbb{Z} / 2 \mathbb{Z}$, and consider Heegaard Floer homology with coefficients in $\mathbb{F}$. Then, there is an isomorphism of graded $\mathbb{F}[U]$-modules

$$
H F_{\text {red }, *}^{+}(Y ; \mathbb{F}) \cong \bigoplus_{1 \leq s \leq g-1}\left(X_{*-s^{2}+1}(g, g-1-s) \oplus X_{*-s^{2}+1}(g, g-1-s)\right) .
$$

Proof Note that $\operatorname{Spin}^{c}(Y) \cong \mathbb{Z}^{2 g}$. However, all of the homology of $Y$ is represented by embedded tori, and hence the adjunction inequality (cf [15, Theorem 8.1]) ensures that $\mathrm{HF}^{+}(Y, \mathfrak{t})$ is non-trivial only for the $\operatorname{Spin}^{c}$ structure $\mathfrak{t}$ with trivial first Chern class. Now, we apply Theorem 4.10 to $n=1$ surgery on the Borromean knot in $\#^{2 g}\left(S^{2} \times S^{1}\right)$, to see that $H F^{+}(Y, \mathfrak{t})$ is isomorphic to the homology of the mapping cone $\mathbb{X}_{\mathfrak{t}}^{+}(1)$. This is particularly simple to do, since all the internal differentials in $A_{S}^{+}$ and $B_{s}^{+}$can be taken to vanish, and hence, we are left with calculating the kernel and cokernels of $D_{1}^{+}$. To simplify notation, we write simply $\mathbb{X}^{+}$for $\mathbb{X}_{\mathfrak{t}}^{+}(1)$. In fact, we consider the reductions of all of these complexes modulo 2, which we suppress from the notation; for example, writing simply $A_{S}^{+}$when we mean that this should be taken modulo 2 , ie $A_{s}^{+} \otimes \mathbb{Z} / 2 \mathbb{Z}$.

Observe that for all $s \geq 0$, the map $v_{s}^{+}$resp. $h_{-s}^{+}$is surjective; and we denote its kernel by $K_{s}$ resp. $K_{-s}$. Indeed, if $|s|$ is sufficiently large, then $K_{s}=0$.

There is a chain map $\Pi_{0}: \mathbb{X}^{+} \longrightarrow A_{0}^{+}$. We claim that this defines a surjection on homology. Specifically, any element $a_{0} \in A_{0}^{+}$can be extended inductively to a sequence $\left\{a_{s}\right\}_{s \in \mathbb{Z}}=a \in \operatorname{Ker} D_{1}^{+}$as follows: if $s \geq 0$, choose $a_{s+1}$ so that $v^{+}\left(a_{s+1}\right)=-h^{+}\left(a_{s}\right)$, and $a_{-s-1}$ so that $h^{+}\left(a_{-s-1}\right)=-v^{+}\left(a_{-s}\right)$. This can be done since $v_{s}^{+}$and $h_{-s}^{+}$are surjective if $s \geq 0$. Note also that the sequence has finite support.

Indeed, we claim that the projection $\Pi_{0}$ induces an isomorphism between the image of the $U^{d}$ on $H_{*}\left(\mathbb{X}^{+}\right)$for all sufficiently large $d$, and $A_{0}^{+}$,

$$
\left(U^{d} H_{*}\left(\mathbb{X}^{+}\right) \subset H_{*}\left(\mathbb{X}^{+}\right)\right) \stackrel{\cong}{\longrightarrow} A_{0}^{+} .
$$

This can be seen as follows. Observe that in all sufficiently large degrees (ie with respect to the integral grading on $\mathbb{X}^{+}$), we have that for all $s>0$, both

$$
v_{*}^{+}: A_{s}^{+} \longrightarrow B_{s}^{+} \text {and } h_{*}^{+}: A_{-s}^{+} \longrightarrow B_{-s+1}^{+}
$$

are injective. It follows at once that all homogeneous elements of $H_{*}\left(\mathbb{X}^{+}\right)$in sufficiently large degrees have non-trivial component in $A_{0}^{+}$. Suppose now that $a$ is such a homology class, and $a_{s}$ denotes its component in $A_{s}^{+}$, so that $a_{0}$ is supported in 
$\bar{C}\{i, j\}$. Then it is a straightforward consequence of Proposition 5.2 and (26) that if $L_{A_{0}^{+}}\left(a_{0}\right)=\max (i, j)$, then for any positive $k \geq 0$,

$$
L_{A_{s}^{+}}\left(a_{s}\right)= \begin{cases}\max \left(i-\frac{s(s-1)}{2}, j-\frac{s(s+1)}{2}\right) & \text { if } s \text { is even } \\ \max \left(j-\frac{s(s-1)}{2}, i-\frac{s(s+1)}{2}\right) & \text { if } s \text { is odd }\end{cases}
$$

In particular, $L\left(a_{s}\right) \leq L\left(a_{0}\right)$ for all $s$ and hence $L\left(a_{0}\right)=L(a)$. It follows at once that projection $\Pi_{0}$ induces an injective map from $U^{d} H_{*}\left(\mathbb{X}^{+}\right) \subset H_{*}\left(\mathbb{X}^{+}\right) \longrightarrow A_{0}^{+}$. It is straightforward now to see that the map $U: A_{0}^{+} \longrightarrow A_{0}^{+}$is surjective. This then verifies the isomorphism from (27).

Thus, it follows that for all sufficiently large $d$,

$$
\left.\frac{H_{*}\left(\mathbb{X}^{+}\right)}{U^{d} \cdot H_{*}\left(\mathbb{X}^{+}\right)} \cong \operatorname{Ker} \Pi_{0}\right|_{H_{*}\left(\mathbb{X}^{+}\right)} .
$$

Now, for integers $i \geq 0$, let $\mathbb{X}_{|\cdot| \leq i}^{+}$denote the quotient complex of $\mathbb{X}^{+}$generated by $A_{s}^{+}$with $-i \leq s \leq i$ and $B_{s}^{+}$with $-i+1 \leq s \leq i$. We have projection maps

$$
\Pi_{|\cdot| \leq i}: \mathbb{X}^{+} \longrightarrow \mathbb{X}_{|\cdot| \leq i}^{+}
$$

which, when $i=0$, coincides with the projection $\Pi_{0}$ considered earlier. Letting $F_{i}=\operatorname{Ker} \Pi_{|\cdot| \leq i}$, we have

$$
F_{0} \supseteq F_{1} \supseteq F_{2} \supseteq F_{3} \supseteq \cdots \supseteq F_{i} \supseteq F_{i+1} \cdots
$$

Moreover, we claim that for all $s \geq 1$, there is a short exact sequence

$$
0 \longrightarrow H_{*}\left(F_{S}\right) \longrightarrow H_{*}\left(F_{s-1}\right) \stackrel{\pi}{\longrightarrow} K_{s} \oplus K_{-s} \longrightarrow 0
$$

To see this, note that the restriction of $\Pi_{|\cdot| \leq s}$ to $F_{s-1}$ naturally induces a short exact sequence

$$
0 \longrightarrow F_{s} \longrightarrow F_{s-1} \longrightarrow M\left(v_{s}^{+} \oplus h_{-s}^{+}: A_{s}^{+} \oplus A_{-s}^{+} \longrightarrow B_{s}^{+} \oplus B_{-s+1}^{+}\right) \longrightarrow 0,
$$

and it is easy to see that the homology of the mapping cone of $v_{s}^{+} \oplus h_{-s}^{+}$is identified with $K_{s} \oplus K_{-s}$. It remains to show that the map from $H_{*}\left(F_{s-1}\right) \longrightarrow K_{s} \oplus K_{-s}$ is surjective. But this follows at once from the fact that for all $t>s, v_{t}^{+}: A_{t}^{+} \longrightarrow B_{t}^{+}$and $h_{-t}^{+}: A_{-t}^{+} \longrightarrow B_{-t+1}^{+}$are surjective. For instance, given $a_{s} \in K_{s}$ with $s>0$, we can use the surjectivity of $v_{k}^{+}$for all $k>0$ to inductively define a sequence $\left\{a_{k} \in A_{k}^{+}\right\}_{k \in \mathbb{Z}}$ 
by

$$
a_{k}= \begin{cases}0 & \text { if } k<s \\ a_{s} & \text { if } k=s \\ -R_{k} \circ h_{k-1}^{+}\left(a_{k-1}\right) & \text { if } k>s,\end{cases}
$$

where $R_{k}: B_{k}^{+} \longrightarrow A_{k}^{+}$is a right inverse for the map $v_{k}^{+}: A_{k}^{+} \longrightarrow B_{k}^{+}$(for $k \geq 0$ ) viewed as a $\mathbb{F}$-module map. This element determines a cycle in $F_{s-1} \subset \mathbb{X}^{+}$whose restriction to $A_{s}$ is the given element $a_{s} \in K_{s}$. An element $a_{-s} \in K_{-s}$ can be similarly extended to a sequence $\left\{a_{k}\right\}_{k \in \mathbb{Z}}$ supported in $a_{k}$ for $k \leq-s$, by switching the roles of $v^{+}$and $h^{+}$. From the above description of $C$ for the Borromean knot, it follows that $K_{s} \cong X(g, g-1-s)$. Indeed, with respect to the induced grading from $\mathbb{X}^{+}$, it is easy to see that $K_{s} \cong X_{*-s^{2}+1}(g, g-1-s)$. Combining this with the exact sequence of (29), we have proved the isomorphism claimed in the theorem as $\mathbb{F}$-modules.

In effect, the sequence of elements $\left\{a_{k}\right\}_{k \in \mathbb{Z}}$ defined above gives a right inverse to the map $\pi$ in (29). To verify the theorem on the level of $\mathbb{F}[U]$-modules, it suffices to show that a careful choice of elements $\left\{a_{k}\right\}_{k \in \mathbb{Z}}$ provides a $\mathbb{Z}[U]$-equivariant splitting of $\pi$. Since $K_{s}=\bar{C}\{i<0, j \geq s\}$, in view of Proposition 5.2, the image of $h^{+}\left(K_{s}\right)$ is $\bar{C}\{i \geq 0, j<-s\}$. Now, the inclusion map of $\bar{C}\{i \geq 0\} \longrightarrow \bar{C}\{\max (i, j-s-1) \geq 0\}$ provides a right inverse $R_{s}$ to $v_{s}^{+}$which, of course, is not a $\mathbb{Z}[U]$-module map. However, it is easy to see that its restriction to $\bar{C}\{i \geq 0, j<-s\}$ is $\mathbb{Z}[U]$-equivariant. Moreover, this image is contained in the kernel of $h_{k+1}^{+}$. Thus, the sequence of elements $\left\{a_{k}\right\}_{k \in \mathbb{Z}}$ constructed from $a_{s}$ using this right inverse has at most two nonzero elements, and it is clearly a $\mathbb{Z}[U]$-equivariant splitting. When $s<0$, we use an analogous right inverse for $h_{s}^{+}$, to complete the $\mathbb{F}[U]$-module splitting of short exact sequence (29). This completes the proof of the theorem.

It is suggestive to compare the above result with Seiberg-Witten theory over circle bundles, compare Mrowka-Ozsváth-Yu [9] and Nicolaescu [10]; see also KronheimerMrowka [6] for a construction of the Floer theory in this context. Results from [9] and [10] show that the moduli space of irreducible solutions to the Seiberg-Witten equations are identified with a disjoint union of symmetric products of the underlying Riemann surface,

$$
\left.\left.\coprod_{1 \leq s \leq g-1}\left(\operatorname{Sym}^{g-1-s}(\Sigma)\right) \bigsqcup \operatorname{Sym}^{g-1-s}(\Sigma)\right)\right) .
$$

Theorem 5.5 can be thought of as an algebraic reflection of this geometric phenomenon.

According to the following result, increasing the Euler number $n$ of the circle bundle, causes the total rank of $H F_{\text {red }}^{+}$to drop. This corresponds, in gauge theory, to the 
existence of flow-lines which connect the vortex moduli spaces with the Jacobian torus, compare Ozsváth-Szabó [11].

Let $Y(g, n)$ denote the Euler number $n$ circle bundle over an oriented two-manifold of genus $g$. In this case, $\operatorname{Spin}^{c}(Y(g, n)) \cong \mathbb{Z}^{2 g} \oplus \mathbb{Z} / n \mathbb{Z}$. By the adjunction inequality, however, only those $\operatorname{Spin}^{c}$ structures whose first Chern class is torsion have non-trivial $H F^{+}$. Indeed, realizing $Y(g, n)$ as $+n$-surgery on the Borromean knot induces an identification of $\mathbb{Z} / n \mathbb{Z}$ with these $\operatorname{Spin}^{c}$ structures.

We have the following generalization of Theorem 5.5 which allows us to calculate the Heegaard Floer homology for any non-trivial circle bundle over an oriented twomanifold. Note that the hypothesis is that the Euler number $n>0$; but the general case follows at once since $-Y(g, n) \cong Y(g,-n)$, and hence, by the behaviour of Heegaard Floer homology under orientation reversal, cf [16], we see that

$$
H F_{\text {red, } k}^{+}(Y(g,-n), i) \cong H F_{\text {red, }-k}^{+}(Y(g,-n), i) .
$$

Theorem 5.6 Let $Y(g, n)$ denote the circle bundle with Euler number $n>0$ over a surface of genus $g$. Then, for any choice of $[i] \in \mathbb{Z} / n \mathbb{Z}$, let $j$ be an integer with minimal absolute value among all integers congruent to $i(\bmod n)$. We have that

$$
H F_{\text {red,* }}^{+}(Y(g, n), i ; \mathbb{Z} / 2 \mathbb{Z}) \cong \bigoplus_{\{s \equiv i \quad(\bmod n) \mid s \neq j\}} X_{c(i, s)}(g, g-1-|s|),
$$

where here

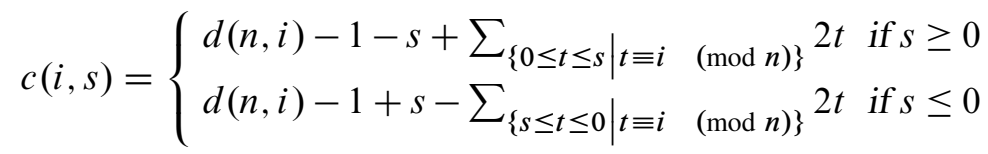

Proof This is a straightforward adaptation of the proof of Theorem 5.5 above.

\section{References}

[1] D Bar-Natan, On Khovanov's categorification of the Jones polynomial, Algebr. Geom. Topol. 2 (2002) 337-370 MR1917056

[2] O Collin, B Steer, Instanton Floer homology for knots via 3-orbifolds, J. Differential Geom. 51 (1999) 149-202 MR1703606

[3] S Jabuka, T Mark, On the Heegaard Floer homology of a surface times a circle MR2189929 arXiv:math/0502328

[4] M Khovanov, A categorification of the Jones polynomial, Duke Math. J. 101 (2000) 359-426 MR1740682 
[5] M Khovanov, L Rozansky, Matrix factorizations and link homology arXiv: math.QA/0401268

[6] PB Kronheimer, TS Mrowka, Floer homology for Seiberg-Witten Monopoles, preprint

[7] E S Lee, The support of the Khovanov's invariants for alternating knots (2002) arXiv: math.GT/0201105

[8] I G Macdonald, Symmetric products of an algebraic curve, Topology 1 (1962) 319-343 MR0151460

[9] T Mrowka, P Ozsváth, B Yu, Seiberg-Witten monopoles on Seifert fibered spaces, Comm. Anal. Geom. 5 (1997) 685-791 MR1611061

[10] L I Nicolaescu, Adiabatic limits of the Seiberg-Witten equations on Seifert manifolds, Comm. Anal. Geom. 6 (1998) 331-392 MR1651420

[11] P Ozsváth, Z Szabó, On embedding circle-bundles in four-manifolds, Math. Res. Lett. 7 (2000) 657-669 MR1809292

[12] P Ozsváth, Z Szabó, Absolutely graded Floer homologies and intersection forms for four-manifolds with boundary, Adv. Math. 173 (2003) 179-261 MR1957829

[13] P Ozsváth, Z Szabó, On the Floer homology of plumbed three-manifolds, Geom. Topol. 7 (2003) 185-224 MR1988284

[14] P Ozsváth, Z Szabó, Holomorphic disks and knot invariants, Adv. Math. 186 (2004) 58-116 MR2065507

[15] P Ozsváth, Z Szabó, Holomorphic disks and three-manifold invariants: properties and applications, Ann. of Math. (2) 159 (2004) 1159-1245 MR2113020

[16] P Ozsváth, Z Szabó, Holomorphic disks and topological invariants for closed threemanifolds, Ann. of Math. (2) 159 (2004) 1027-1158 MR2113019

[17] PS Ozsváth, Z Szabó, Knot Floer homology and rational surgeries (2005) arXiv: math.GT/0504404

[18] P Ozsváth, Z Szabó, On knot Floer homology and lens space surgeries, Topology 44 (2005) 1281-1300 MR2168576

[19] P Ozsváth, Z Szabó, On the Heegaard Floer homology of branched double-covers, Adv. Math. 194 (2005) 1-33 MR2141852

[20] P Ozsváth, Z Szabó, Holomorphic triangles and invariants for smooth four-manifolds, Adv. Math. 202 (2006) 326-400 MR2222356

[21] J A Rasmussen, Floer homology and knot complements, $\mathrm{PhD}$ thesis, Harvard University (2003)

[22] J A Rasmussen, Khovanov homology and the slice genus (2004) arXiv: math.GT/0402131 
Department of Mathematics, Columbia University, New York 1002, USA

Department of Mathematics, Princeton University, New Jersey 08544, USA

petero@math.columbia.edu, szabo@math.princeton.edu

Received: 29 April 2005 Revised: 27 December 2006

Algebraic 83 Geometric Topology, Volume 8 (2008) 